\title{
Bayesian Inference for Nonlinear Multivariate Diffusion Models Observed with Error
}

\author{
A. Golightly ${ }^{\text {a,* }}$ D.J. Wilkinson ${ }^{a}$ \\ ${ }^{a}$ University of Newcastle Upon Tyne, School of Mathematics and Statistics, Merz \\ Court, Newcastle Upon Tyne, NE1 7RU, UK
}

\begin{abstract}
Diffusion processes governed by stochastic differential equations (SDEs) are a well established tool for modelling continuous time data from a wide range of areas. Consequently, techniques have been developed to estimate diffusion parameters from partial and discrete observations. Likelihood based inference can be problematic as closed form transition densities are rarely available. One widely used solution involves the introduction of latent data points between every pair of observations to allow an Euler-Maruyama approximation of the true transition densities to become accurate. In recent literature, Markov chain Monte Carlo (MCMC) methods have been used to sample the posterior distribution of latent data and model parameters; however, naive schemes suffer from a mixing problem that worsens with the degree of augmentation. In this paper, we explore an MCMC scheme whose performance is not adversely affected by the number of latent values. We illustrate the methodology by estimating parameters governing an auto-regulatory gene network, using partial and discrete data that is subject to measurement error.
\end{abstract}

Key words: Bayesian inference, particle filter, MCMC, nonlinear stochastic differential equation

\section{Introduction}

In the last twenty years, the desire to include unpredictable factors into modelling has encouraged much interest in diffusion models. As a consequence, the problem of how to efficiently estimate parameters governing stochastic

\footnotetext{
* Corresponding author.

Email addresses: a.golightly@ncl.ac.uk (A. Golightly), d.j.wilkinson@ncl.ac.uk (D.J. Wilkinson).
} 
differential equations (SDEs) has arisen. Some significant examples where this occurs include economics and finance (Black and Scholes, 1973; Cox et al., 1985) and, more recently, bioinformatics (Gillespie, 1992; Arkin et al., 1998; McAdams and Arkin, 1999).

Since transition densities of diffusions are unavailable in closed form for all but a few special cases, likelihood inference is not trivial. Attempts to overcome this problem can be categorised into three main areas; alternative estimators to the MLE include the use of estimating functions (Bibby and Sørensen, 1995) and the method of moments (Chan et al., 1992). Simulated maximum likelihood estimation (SMLE) approaches have been proposed by Pedersen (1995) and refined by Durham and Gallant (2002). Finally, Bayesian imputation methods have been proposed by Elerian et al. (2001), Roberts and Stramer (2001) and Eraker (2001). Most Bayesian and likelihood approaches approximate the underlying, true transition densities with a first order Euler discretisation. As inter-observation times are usually too large to be used as a time step with the Euler method, the observed low-frequency data are augmented with the introduction of $m-1$ latent data points between every pair of observations. The Gibbs sampler or other Markov chain Monte Carlo (MCMC) schemes can then be used to sample the posterior of latent data and model parameters.

Unfortunately, the most natural Bayesian imputation approach can break down when the amount of augmentation is large. It is well documented (see for example Papaspiliopolous et al. (2003)) that high dependence between unknown parameters in the diffusion (or volatility) coefficient and the missing data can result in arbitrarily slow rates of convergence of naive sampling strategies such as the single site Gibbs sampler employed by Eraker (2001) and Golightly and Wilkinson (2005a). Some attempts have been made to rectify this problem; Roberts and Stramer (2001) transform the SDE to give a constant diffusion coefficient and by so doing are able to overcome the dependence problem. Beskos et al. (2006) propose Monte Carlo methods which are both exact and computionally efficient, but also rely on a transformation to constant volatility. Such transformations can not be applied to many non-linear multivariate diffusions (such as those considered here) (Wilkinson, 2003) and work in this area remains of great interest.

In this paper we examine a Gibbs sampling algorithm that does not break down for large $m$ (but in fact improves) and we compare it to the sequential MCMC scheme of Golightly and Wilkinson (2005b) and Golightly and Wilkinson (2006). Both algorithms are applied to noisy data generated from a simple auto-regulatory gene network; such networks are examined in Golightly and Wilkinson (2005a) and Golightly and Wilkinson (2006) and typically induce a diffusion that is high dimensional and nonlinear. 
The remainder of this paper is organised as follows. In Section 2 we formulate the model and describe the estimation framework; convergence issues of naive sampling strategies are discussed in Section 2.1. We describe a novel MCMC scheme in Section 3 and outline filtering strategies in Section 4. The methodology is illustrated in Section 5 before conclusions are drawn in Section 6 .

\section{Diffusion Models}

We consider inference for a $d$-dimensional Itô Diffusion that satisfies a stochastic differential equation of the form

$$
d Y(t)=\mu(Y(t), \Theta) d t+\beta^{\frac{1}{2}}(Y(t), \Theta) d W(t),
$$

and assume that the conditions under which the SDE can be solved for $Y(t)$ are satisfied.

Often, we will have only partial data and therefore $Y(t)$ will consist of both observable and unobservable components. Hence we define $Y(t)=(X(t), Z(t))^{\prime}$, where $X(t)$ defines the observable part and $Z(t)$ the unobservable part of the system. Note that $X(t)$ and $Z(t)$ have dimensions $d_{1}$ and $d_{2}$ respectively such that $Y(t)$ has dimension $d=d_{1}+d_{2}$. We assume further that the process $X(t)$ is subject to measurement error such that we actually observe

$$
V(t)=X(t)+\epsilon(t)
$$

where $\epsilon(t) \sim \mathrm{N}(0, \Sigma), \Sigma=\operatorname{diag}\left\{\sigma_{i}^{2}\right\}$ for $i=1, \ldots, d_{1}$, represents an independent noise process. Note that for unknown $\Sigma$, we have $\Theta=\left(\theta_{1}, \ldots, \theta_{p}, \sigma_{1} \ldots, \sigma_{d_{1}}\right)^{\prime}$. The assumption of a diagonal $\Sigma$ is in no way essential, but is adopted here in order to simplify the presentation. The process $V(t)$ will be observed at a finite number of times and the objective is to conduct inference for the (unknown) parameter vector $\Theta$ on the basis of these noisy, partial and discrete observations.

As already stated, we work with the discretized version of (1), given by the Euler approximation,

$$
\Delta Y(t)=\mu(Y(t), \Theta) \Delta t+\beta^{\frac{1}{2}}(Y(t), \Theta) \Delta W(t),
$$

where $\Delta W(t)$ is a $d$ dimensional iid $\mathrm{N}(0, I \Delta t)$ random vector. Now suppose we have measurements $v\left(\tau_{i}\right)$ at evenly spaced times $\tau_{0}, \tau_{1}, \ldots, \tau_{T}$ with intervals of length $\Delta^{*}=\tau_{i+1}-\tau_{i}$. As $\Delta^{*}$ is often too large to be used as a time step in (3), we put $\Delta t=\Delta^{*} / m$ for some positive integer $m>1$. By choosing $m$ to be sufficiently large, we can ensure that the discretization bias is arbitrarily 
small, but this also introduces the problem of $m-1$ missing values in between every pair of observations.

We deal with these missing values by dividing the entire time interval $\left[\tau_{0}, \tau_{T}\right]$ into $m T+1$ equidistant points $\tau_{0}=t_{0}<t_{1}<\ldots<t_{n}=\tau_{T}$ such that $V(t)$ is observed at times $t_{0}, t_{m}, \ldots, t_{n}$. Altogether we have $d(T m+1)$ missing values which we substitute with simulations $Y\left(t_{i}\right)$. We refer to the collection of simulated data as the augmented data. Eraker (2001) denotes by $\hat{Y}$ the $d \times(n+1)$ matrix obtained by stacking all elements of the augmented data, that is

$$
\hat{\mathrm{Y}}=\left(\begin{array}{ccccccc}
X_{1}\left(t_{0}\right) & X_{1}\left(t_{1}\right) & \cdots & X_{1}\left(t_{m}\right) & X_{1}\left(t_{m+1}\right) & \cdots & X_{1}\left(t_{n}\right) \\
X_{2}\left(t_{0}\right) & X_{2}\left(t_{1}\right) & \cdots & X_{2}\left(t_{m}\right) & X_{2}\left(t_{m+1}\right) & \cdots & X_{2}\left(t_{n}\right) \\
\vdots & \vdots & & \vdots & \vdots & & \vdots \\
X_{d_{1}}\left(t_{0}\right) & X_{d_{1}}\left(t_{1}\right) & \cdots & X_{d_{1}}\left(t_{m}\right) & X_{d_{1}}\left(t_{m+1}\right) & \cdots & X_{d_{1}}\left(t_{n}\right) \\
Z_{1}\left(t_{0}\right) & Z_{1}\left(t_{1}\right) & \cdots & Z_{1}\left(t_{m}\right) & Z_{1}\left(t_{m+1}\right) & \cdots & Z_{1}\left(t_{n}\right) \\
\vdots & \vdots & & \vdots & \vdots & & \vdots \\
Z_{d_{2}}\left(t_{0}\right) & Z_{d_{2}}\left(t_{1}\right) & \cdots & Z_{d_{2}}\left(t_{m}\right) & Z_{d_{2}}\left(t_{m+1}\right) & \cdots & Z_{d_{2}}\left(t_{n}\right)
\end{array}\right) .
$$

We now let $Y^{i}=\left(X^{i}, Z^{i}\right)$ denote the $i^{\text {th }}$ column of $\hat{Y}$. By adopting a fully Bayesian approach, we summarise our a priori beliefs about $\Theta$ and $Y^{0}$ via the prior distributions $\pi(\Theta)$ and $\pi\left(Y^{0}\right)$ respectively. Then the joint posterior density for parameters and augmented data is given by

$$
\pi\left(\hat{\mathrm{Y}}, \Theta \mid D_{n}\right) \propto \pi(\Theta) \pi\left(Y^{0}\right)\left[\prod_{i=0}^{n-1} \pi\left(Y^{i+1} \mid Y^{i}, \Theta\right)\right]\left[\prod_{i \in\{0, m, \ldots, n\}} \pi\left(v^{i} \mid X^{i}, \Theta\right)\right]
$$

where $v^{i}$ denotes $v\left(t_{i}\right), D_{n}=\left(v^{0}, v^{m}, \ldots v^{n}\right)$,

$$
\pi\left(Y^{i+1} \mid Y^{i}, \Theta\right)=\phi\left(Y^{i+1} ; Y^{i}+\mu_{i} \Delta t, \beta_{i} \Delta t\right)
$$

and

$$
\pi\left(v^{i} \mid X^{i}, \Theta\right)=\phi\left(v^{i} ; X^{i}, \Sigma\right) .
$$

Here, $\mu_{i}=\mu\left(Y^{i}, \Theta\right), \beta_{i}=\beta\left(Y^{i}, \Theta\right)$ and $\phi(\cdot ; \psi, \gamma)$ denotes the Gaussian density with mean $\psi$ and variance matrix $\gamma$. Note that $\pi\left(Y^{i+1} \mid Y^{i}, \Theta\right)$ is the one step ahead transition density obtained from the Euler discretization.

\subsection{Gibbs Sampling (Convergence Issues)}

As discussed in Tanner and Wong (1987), inference may proceed by alternating between simulation of parameters conditional on augmented data, and 
simulation of the missing data given the observed data and the current state of the model parameters. As the joint posterior, (4) is usually high dimensional, a Gibbs sampler is a particularly convenient way of sampling from it. For nonlinear diffusions, direct sampling of the full conditional distributions is not possible and a Metropolis within Gibbs move can be implemented. Various sampling strategies have been proposed; Eraker (2001) and Golightly and Wilkinson (2005a) update one column of $\hat{Y}$ at a time whilst Shephard and Pitt (1997) update $\hat{Y}$ in random blocks. The former method of updating one column (which we refer to as the "single site Gibbs sampler") leads to an algorithm with a rate of convergence that worsens linearly with $m$. In fact, as $m$ increases, one can make very precise inference about the diffusion coefficient of the process via the quadratic variation (see Roberts and Stramer (2001)). It is this dependence (between the quadratic variation and diffusion coefficient) that results in slow mixing of MCMC algorithms such as the single site Gibbs sampler. Though the block updating method of Shephard and Pitt (1997) is able to help overcome the dependence within the latent process, dependence between the parameters and latent process remains high and covergence will still become arbitrarily slow as either $m$ or the number of observations increases. Roberts and Stramer (2001) overcome this dependence in the context of univariate diffusions by transforming the missing data, giving a partially non-centered parameterisation which leads to an irreducible algorithm even in the limit $m \rightarrow \infty$. However, for a $d$-dimensional diffusion satisfying (1), finding such a transformation requires an invertible function, $g: \mathbf{R}^{d} \rightarrow \mathbf{R}^{d}$ such that

$$
\nabla g(\nabla g)^{\prime}=\beta^{-1}
$$

As discussed by Papaspiliopolous et al. (2003), this equation is almost always impossible to solve in practice for general non-linear multivariate diffusions such as those considered here. Sequential filtering algorithms have been suggested in Golightly and Wilkinson (2005b) among others and rely on a joint update of $\Theta$ and the latent data. Whilst such schemes overcome the dependence issue, they are known to suffer from degeneracy problems as the amount of data increases (see Liu and West (2001) for a discussion).

Here, we develop and illustrate an MCMC strategy which can be easily implemented for any nonlinear multivariate diffusion and does not suffer from the convergence problems of the single site Gibbs sampler; in essence, by alternatively sampling from the posterior of parameters and the driving Brownian motion process (rather than the latent data), we can overcome the dependence between $\Theta$ and the latent data. The idea is motivated by the "innovation" scheme of Chib et al. (2004); however, our details and strategy are different; the algorithm we consider can be applied to any partially observed diffusion process (that may be subject to measurement error) and doesn't rely on being able to untangle the diffusion - that is for our method, the unobserved component, $Z(t)$ can depend on $X(t)$ in a nonlinear way (and vice-versa). We also compare the algorithm to the simulation filter of Golightly and Wilkinson 
(2006) — see Section 4 for a brief outline.

\section{The Innovation Scheme}

Consider data $D_{n}=\left(v^{0}, v^{m}, \ldots, v^{n}\right)$ and a Gibbs sampler which samples from the distribution $\left(\Theta, \hat{Y} \mid D_{n}\right)$ by alternating between draws of $\Theta$ conditional on $\hat{\mathrm{Y}}$ (and $D_{n}$ ) and the latent data, $\hat{\mathrm{Y}}$, conditional on $\Theta$ (and $D_{n}$ ). Naturally, for large $m$ such a strategy will result in a chain with arbitrarily slow convergence times. The innovation scheme, however, is subtly different.

Corresponding to the sample path of $Y(t)$, given by $\hat{Y}=\left(Y^{0}, Y^{1}, \ldots, Y^{n}\right)$, is a skeleton of $W(t)$, the driving Brownian process. We denote this skeleton by $\hat{\mathrm{W}}=\left(W^{0}, W^{1}, \ldots, W^{n}\right)$ and note that under the Euler approximation, (3) (or any discrete approximation of (1)), there is a one-to-one relationship between $\hat{W}$ and $\hat{Y}$, conditional on $\Theta$. Therefore, rather than sample the distribution $\left(\Theta, \hat{\mathrm{Y}} \mid D_{n}\right)$, the innovation scheme samples $\left(\Theta, \hat{\mathrm{W}} \mid D_{n}\right)$ by alternating between draws of $\Theta$ conditional on the data and $\hat{W}$, and $\hat{W}$ conditional on $\Theta$ and the data.

Algorithmically:-

(1) Initialise all unknowns. Set the iteration counter to $s=1$.

(2) Sample $\hat{W}$ conditional on parameters and the data by updating the latent data, using $\left(\hat{\mathrm{Y}} \mid \Theta, D_{n}\right)$.

(3) Update parameters by drawing from $\left(\Theta \mid \hat{\mathrm{W}}, D_{n}\right)$.

(4) Increment $s$ and return to 2.

Step 2 can be performed using a series of Metropolis-Hastings (M-H) moves (see Section 3.1). By updating the latent data, $\hat{W}$ is obtained deterministically, conditional on the current value of $\Theta$. We use a $\mathrm{M}-\mathrm{H}$ step in 3 to obtain a new $\Theta$ (see Section 3.2). Our new value of $\Theta$ can then be combined with the skeleton, $\hat{W}$ to give a new sample path $\hat{Y}$, ready to be used in step 2 . Hence at every iteration of the algorithm, the volatility of the sample path will be consistent with the current parameter value - this is crucial in order to overcome the dependence issue highlighted by Roberts and Stramer (2001). We now detail each step individually.

\subsection{Updating the latent data points}

Whilst Chib et al. (2004) update $\hat{Y}$ using random block sizes, our algorithm requires updating in blocks of size $2 m+1$. Note that in the case of complete observation and the absence of measurement error, there is a much simpler 
version of this algorithm using blocks of size $m$, but we do not present that here.

Consider times $t_{j}, t_{M}$ and $t_{M^{+}}$where $j$ is an integer multiple of $m, M=$ $j+m$ and $M^{+}=M+m$. Note that these times correspond to the noisy and partial observations, $v^{j}, v^{M}$ and $v^{M^{+}}$. Treating $Y^{j}$ and $Y^{M^{+}}$as fixed, the full conditional for $Y^{j+1}, \ldots, Y^{j+2 m-1}$ is

$$
\pi\left(Y^{j+1}, \ldots, Y^{j+2 m-1} \mid Y^{j}, Y^{M^{+}}, v^{M}, \Theta\right) \propto \pi\left(v^{M} \mid X^{M}, \Theta\right) \prod_{i=j}^{j+2 m-1} \pi\left(Y^{i+1} \mid Y^{i}, \Theta\right) .
$$

By sampling this distribution for $j=0, m, \ldots n-2 m$, the use of overlapping blocks with a free mid-point ensures that we have an irreducible algorithm. Hence at iteration $s$ of the innovation scheme, we would like to draw

$$
Y^{j+1}, \ldots, Y^{j+2 m-1} \sim \pi\left(Y^{j+1}, \ldots, Y^{j+2 m-1} \mid Y^{j}, Y^{M^{+}}, v^{M}, \Theta\right)
$$

where $Y^{j}$ is obtained at iteration $(s)$ and $Y^{M^{+}}$at iteration $(s-1)$. Naturally, the end-points of the process, $Y^{0}$ and $Y^{n}$ must also be updated. For every sweep of the sampler we fix $Y^{m}$ and draw from

$$
\pi\left(Y^{0}, \ldots, Y^{m-1} \mid Y^{m}, v^{0}, \Theta\right) \propto \pi\left(v^{0} \mid X^{0}, \Theta\right) \prod_{i=0}^{m-1} \pi\left(Y^{i+1} \mid Y^{i}, \Theta\right)
$$

and fix $Y^{n-m}$ and draw from

$$
\pi\left(Y^{n-m+1}, \ldots, Y^{n} \mid Y^{n-m}, v^{n}, \Theta\right) \propto \pi\left(v^{n} \mid X^{n}, \Theta\right) \prod_{i=n-m}^{n-1} \pi\left(Y^{i+1} \mid Y^{i}, \Theta\right),
$$

thus ensuring that $Y^{0}$ and $Y^{n}$ are updated.

Note that simulating $Y^{j+1}, \ldots, Y^{j+2 m-1}$ is not trivial. The Euler scheme allows handling of the likelihood between two consecutive values of the process (since we have a linear Gaussian structure in this case). However, obtaining the conditional density of missing values between two given observations that are $2 m$ steps apart, under the non-linear structure of the underlying diffusion process, is complicated. As discussed in Chib et al. (2004), a similar process (which we denote by $Y_{*}$ ) can be considered as a proposal in an MCMC step — one that starts at $Y_{*}\left(t_{j}\right)=Y^{j}$, ends at $Y_{*}\left(t_{M^{+}}\right)=Y^{M^{+}}$and has the same diffusion function as the $Y$ process. Here, we consider the diffusion bridge of Durham and Gallant (2002) and Chib et al. (2004),

$$
\begin{aligned}
d Y_{*}(t) & =\mu_{*}\left(Y_{*}, \Theta\right) d t+\beta^{\frac{1}{2}}\left(Y_{*}, \Theta\right) d W(t) \\
& =\frac{Y_{*}\left(t_{M^{+}}\right)-Y_{*}(t)}{t_{M+}-t} d t+\beta^{\frac{1}{2}}\left(Y_{*}, \Theta\right) d W(t),
\end{aligned}
$$


and suppress the dependence of $\mu_{*}$ on $t$ to simplify the notation. Note that (10) can be easily sampled using the Euler approximation and numerical simulation is discussed in Section 3.1.1. We now let $P_{Y \mid \Theta}$ denote the law of the process given by (1) and $Q_{Y \mid \Theta}$ denote the law of the process, (10). Since $d Y(t)$ and $d Y_{*}(t)$ have common diffusion functions, $P_{Y \mid \Theta}$ and $Q_{Y \mid \Theta}$ are locally equivalent and the resulting likelihood ratio for the path $\left\{Y(t): t \in\left[t_{j}, t_{M+}\right]\right\}$ is given by Girsanov's formula (Øksendal, 1995, p. 162). Ignoring dependence on $\Theta$, we obtain

$$
\begin{aligned}
\log \left\{\frac{d P}{d Q}(Y(t))\right\} & =\text { const }+\int_{t_{j}}^{t_{M^{+}}}\left[\mu(Y)-\mu_{*}(Y)\right]^{\prime} \beta^{-1}(Y) d Y(t) \\
& -\frac{1}{2} \int_{t_{j}}^{t_{M^{+}}}\left[\mu(Y)-\mu_{*}(Y)\right]^{\prime} \beta^{-1}(Y)\left[\mu(Y)-\mu_{*}(Y)\right] d t .
\end{aligned}
$$

Hence, at iteration $s$ of the innovation scheme we can propose the path $Y_{*}^{j+1}, \ldots, Y_{*}^{M^{+}-1}$ by sampling from (10) and accept it with probability

$$
\min \left\{1, \frac{\frac{d P}{d Q}\left(Y_{*}(t)\right) \pi\left(v^{M} \mid X_{*}^{M}\right)}{\frac{d P}{d Q}(Y(t)) \pi\left(v^{M} \mid X^{M}\right)}\right\} .
$$

\subsubsection{Numerical Simulation}

The proposal process, (10) can easily be sampled by utilising the Euler approximation. Hence, treating $Y^{j}$ and $Y^{M^{+}}$as fixed, we may propose $Y_{*}^{i+1}$, for $i=j, \ldots, M^{+}-2$ using

$$
Y_{*}^{i+1} \mid Y_{*}^{i}, Y^{M^{+}}, \Theta \sim \mathrm{N}\left(Y_{*}^{i}+\frac{Y^{M+}-Y_{*}^{i}}{M^{+}-i}, \beta\left(Y_{*}^{i}, \Theta\right) \Delta t\right) .
$$

Such a proposal however, suffers two major drawbacks. Firstly, for a "small" measurement error variance, not conditioning on the data point $v^{M}$ will lead to a low acceptance rate. Secondly, the variance of (12) is non-anticipating. We therefore use a slightly different two step discrete time approximation of (10) by utilising the modified bridge construct of Durham and Gallant (2002).

Consider first the task of proposing $Y_{*}^{j+1}, \ldots, Y_{*}^{M}$. An efficient method would be to draw $Y_{*}^{i+1}$, for $i=j, \ldots, M-1$ from a Gaussian approximation to $\pi\left(Y_{*}^{i+1} \mid Y_{*}^{i}, v^{M}, Y^{M^{+}}, \Theta\right)$, denoted by $\tilde{\pi}\left(Y_{*}^{i+1} \mid Y_{*}^{i}, v^{M}, Y^{M^{+}}, \Theta\right)$ which we derive in Appendix A.3. We find however that the simpler Gaussian approximation, $\tilde{\pi}\left(Y_{*}^{i+1} \mid Y_{*}^{i}, v^{M}, \Theta\right)$ given by (A.8) works sufficiently well for the model considered in Section 5.

Finally, we draw $Y_{*}^{i+1}$, for $i=M, \ldots, M^{+}-2$ from the Gaussian approx- 
imation, $\tilde{\pi}\left(Y_{*}^{i+1} \mid Y_{*}^{i}, Y^{M^{+}}, \Theta\right)$ given by (A.5). This density was first used in the context of fully observed diffusions with no error by Durham and Gallant (2002) and the path produced by the density is often refered to as the modified bridge. See Appendix (A.1) for a derivation.

If at the end of iteration $s-1$ we have $Y^{j+1}, \ldots, Y^{M^{+}-1}$, then at iteration $s$ we accept a move to $Y_{*}^{j+1}, \ldots, Y_{*}^{M^{+}-1}$ with probability $\min \{1, \alpha\}$ where $\alpha$ is given by

$$
\frac{\left[\pi\left(v^{M} \mid X_{*}^{M}\right) \prod_{i=j}^{M^{+}-1} \pi\left(Y_{*}^{i+1} \mid Y_{*}^{i}\right)\right]\left[\prod_{i=j}^{M-1} \tilde{\pi}\left(Y^{i+1} \mid Y^{i}, v^{M}\right) \prod_{i=M}^{M^{+}-2} \tilde{\pi}\left(Y^{i+1} \mid Y^{i}, Y^{M^{+}}\right)\right]}{\left[\pi\left(v^{M} \mid X^{M}\right) \prod_{i=j}^{M^{+}-1} \pi\left(Y^{i+1} \mid Y^{i}\right)\right]\left[\prod_{i=j}^{M-1} \tilde{\pi}\left(Y_{*}^{i+1} \mid Y_{*}^{i}, v^{M}\right) \prod_{i=M}^{M^{+}-2} \tilde{\pi}\left(Y_{*}^{i+1} \mid Y_{*}^{i}, Y^{M^{+}}\right)\right]}
$$

after suppressing dependence on $\Theta$. Note that we bracket the target and proposal densities for transparency. We repeat this step for $j=0, m, \ldots, n-2 m$ leaving the task of updating $Y^{0}$ and $Y^{n}$. We achieve this by drawing from the densities (8) and (9) using a M-H step. We start by drawing $Y_{*}^{0}$ using a Gaussian random walk type move. We then propose $Y_{*}^{1}, \ldots, Y_{*}^{m-1}$ from (A.5) with $M^{+}$replaced by $m$. Finally, we treat $Y^{n-m}$ as fixed and draw $Y_{*}^{i+1}$ for $i=n-m, \ldots, n-1$, from $\tilde{\pi}\left(Y_{*}^{i+1} \mid Y_{*}^{i}, v^{n}, \Theta\right)$ (see Appendix (A.2)). Acceptance probabilities for these moves can be computed in the usual way.

\subsection{Sampling $\Theta$ conditional on the Brownian process}

The final step in the innovation scheme is to sample a new $\Theta$ conditional on the Brownian process; since we cannot update $\Theta$ directly, we use a M-H step. We first combine the current sample path, $\hat{Y}$ with the current parameter value, $\Theta$ to obtain a skeleton path of the Brownian process, $\hat{W}$. This is valid since, conditional on $\Theta$ there is a one-to-one relationship between $\hat{Y}$ and $\hat{W}$ over $t \in[0, T]$ (Chib et al., 2004). Hence we can obtain $\hat{W}$ (corresponding to the current sample path, $\hat{Y}$ ), for example, by re-arranging the Euler approximation to give the Brownian increments, $\Delta W^{i}=W^{i+1}-W^{i}$ for $i=0, \ldots, n-1$, as a function of the latent data points $Y^{i}, Y^{i+1}$ and $\Theta$. That is

$$
\Delta W^{i}=\left(\beta_{i}\right)^{-\frac{1}{2}}\left(\Delta Y^{i}-\mu_{i} \Delta t\right)
$$

where $\Delta Y^{i}=Y^{i+1}-Y^{i}$ and we use the shorthand notation of Section 2 for $\mu\left(Y^{i}, \Theta\right)$ and $\beta\left(Y^{i}, \Theta\right)$. Note that in this step, $Y^{0}$ and $W^{0}$ are treated as fixed so that $\hat{W}$ is entirely determined from the increments.

We then propose a new $\Theta_{*}$ using a suitable proposal density $g(\cdot)$ which may depend on $Y(t), W(t)$ and the current $\Theta$. We find that the Gaussian random 
walk update of Golightly and Wilkinson (2005a) works well. Then, by combining $\Theta_{*}$ with $\left\{\Delta W^{i}, i=0, \ldots n-1\right\}$ and using the Euler approximation, (3), we can propose a new sample path $Y^{0}, Y_{*}^{1}, \ldots, Y_{*}^{n}$ deterministically. The resulting acceptance probability is analagous to that when performing a joint update of $\Theta$ and the latent data, $\hat{Y}$. Since the new path has been proposed, all be it deterministically, from (3) and $g(\cdot)$ is symmetric in $\Theta$ and $\Theta_{*}$ for the random walk update, the acceptance probability for the move reduces to

$$
\min \left\{1, \frac{\pi\left(\Theta_{*}\right) \prod_{i=1}^{T} \pi\left(v^{i m} \mid X_{*}^{i m}, \Theta_{*}\right)}{\pi(\Theta) \prod_{i=1}^{T} \pi\left(v^{i m} \mid X^{i m}, \Theta\right)}\right\} .
$$

Note that conditional on $\Theta, \Delta W^{i}$ can be obtained from $\Delta Y^{i}$ under any discrete time approximation of the underlying SDE since there is a one-to-one relationship between $\hat{\mathrm{W}}$ and $\hat{\mathrm{Y}}$. Choice of this approximation is important as it is then used to propose a new sample path, $Y^{0}, Y_{*}^{1}, \ldots, Y_{*}^{n}$. In this context, use of the first order Euler discretisation as a proposal density is somewhat inefficient, since using it ignores the data, $v^{0}, v^{m}, \ldots, v^{n}$. We therefore use a slightly different approach by utilising the modified bridge of (A.2).

Consider times $t_{j}$ and $t_{M}$ - recall that $j$ is an integer multiple of $m$ and $M=$ $j+m$. Fixing $Y^{j}$ and for $i=j, j+1 \ldots, M-1$, the density $\tilde{\pi}\left(Y^{i+1} \mid Y^{i}, v^{M}, \Theta\right)$ can be thought of as the transition density of the discrete time process of the form

$$
Y^{i+1}=Y^{i}+\psi_{i} \Delta t+\left(\gamma_{i}\right)^{\frac{1}{2}} \Delta W^{i}, \quad \Delta W^{i} \sim \mathrm{N}_{d}(0, I \Delta t),
$$

where $\psi_{i}$ and $\gamma_{i}$ are given by (A.9) and (A.10) respectively. Note that in the case of complete data and no error, simulating from (14) will give a diffusion bridge conditioned to start at $Y^{j}$ and finish at $Y^{M}$. Our goal then is to use (14) to propose a new path $Y^{0}, Y_{*}^{1}, \ldots, Y_{*}^{n}$ conditional on the proposed new $\Theta_{*}$ and $\hat{W}$. To achieve this, we first calculate the Brownian increments, corresponding to the skeleton path, by re-arranging (14) to give $\Delta W^{i}$ as a function of $\hat{Y}$ and $\Theta$. This gives the one-to-one relationship,

$$
\Delta W^{i}=\left(\gamma_{i}\right)^{-\frac{1}{2}}\left(\Delta Y^{i}-\psi_{i} \Delta t\right)
$$

Now, since $Y^{0}$ and $W^{0}$ are assumed to be fixed, (15) is easily applied for each $j=0, m, \ldots, n-m$ and $i=j, \ldots, M-1$ to give $\left\{\Delta W^{i}, i=0, \ldots n-1\right\}$. We then propose a new $\Theta_{*}$ using a Gaussian random walk move and obtain the proposed new sample path deterministically using $\Theta_{*},\left\{\Delta W^{i}, i=0, \ldots n-1\right\}$ and (14). Note that in the case of full observation with no error, the increments $\Delta W^{i}, i=m-1,2 m-1, \ldots, n-1$, corresponding to the end-points of each subinterval are not stored, as in this case, the latent path is conditioned to hit each data point exactly. 
We accept $\Theta_{*}\left(\right.$ and therefore $\left.Y_{*}^{0}, Y_{*}^{1}, \ldots, Y_{*}^{n}\right)$ with probability $\min \{1, \alpha\}$ where $\alpha$ is given by

$$
\frac{\left[\pi\left(\Theta_{*}\right) \prod_{i=1}^{T} \pi\left(v^{i m} \mid X_{*}^{i m}, \Theta_{*}\right) \prod_{i=0}^{n-1} \pi\left(Y_{*}^{i+1} \mid Y_{*}^{i}, \Theta_{*}\right)\right]\left[\prod_{i=0}^{n-1} \tilde{\pi}\left(Y^{i+1} \mid Y^{i}, v^{(\lfloor i / m+1\rfloor) m}, \Theta\right)\right]}{\left[\pi(\Theta) \prod_{i=1}^{T} \pi\left(v^{i m} \mid X^{i m}, \Theta\right) \prod_{i=0}^{n-1} \pi\left(Y^{i+1} \mid Y^{i}, \Theta\right)\right]\left[\prod_{i=0}^{n-1} \tilde{\pi}\left(Y_{*}^{i+1} \mid Y_{*}^{i}, v^{(\lfloor i / m+1\rfloor) m}, \Theta_{*}\right)\right]}
$$

where $\lfloor x\rfloor$ denotes the integer part of $x$.

After every iteration of the innovation scheme, each new $\Theta$ is entirely consistent with the sample path $\hat{Y}$, and the dependence between them is overcome. For further discussion see Chib et al. (2004).

\section{Sequential Filtering for Parameters and State - the Simulation Filter}

In this section we briefy summarise the the sequential MCMC scheme of Golightly and Wilkinson (2005b) and Golightly and Wilkinson (2006) which we refer to as the simulation filter.

In the context of discrete time series with unobserved state variables, Bayesian sequential filtering has been discussed extensively, e.g., Berzuini et al. (1997), Pitt and Shephard (1999) and Doucet et al. (2000) and Del Moral et al. (2002). Filtering for both parameters and state has been discussed by Liu and West (2001), Stroud et al. (2004) and Johannes et al. (2006). We consider data $D_{j}=\left(v^{0}, v^{m}, \ldots, v^{j}\right)$, (where $j$ is an integer multiple of $m$ ) arriving at times $t_{0}, t_{m} \ldots, t_{j}$. Recall that at time $t_{M}$ (putting $M=j+m$ ), new data $v^{M}$ are accompanied by $m$ missing columns, $Y^{j+1}, \ldots, Y^{M}$. As each observation becomes available we are interested in the on-line estimation of the unknown parameter vector, $\Theta$.

We assume that we have an equally weighted sample of size $S,\left\{\left(\Theta_{(s)}, Y_{(s)}^{j}\right), s=\right.$ $1, \ldots, S\}$ (with weights $w_{(s)}^{j}=1 / S$ ), from the distribution $\pi\left(\Theta, Y^{j} \mid D_{j}\right)$, which we will denote by $\pi_{j}\left(\Theta, Y^{j}\right)$. At time $t_{M}$, we observe $v^{M}$ and assimilation of the information contained in $v^{M}$ consists of generating a sample, $\left\{\left(\Theta_{(s)}, Y_{(s)}^{M}\right), s=\right.$ $1, \ldots, S\}$ from the posterior $\pi_{M}\left(\Theta, Y^{M}\right)$ which can be found by formulating the posterior for parameters and augmented data, then integrating out the 
latent data. Using (4) we have

$$
\pi_{M}\left(\Theta, Y^{M}\right) \propto \int_{\hat{\mathrm{Y}}_{M}} \pi(\Theta) \pi\left(Y^{0}\right) \prod_{i=0}^{M-1} \pi\left(Y^{i+1} \mid Y^{i}, \Theta\right) \prod_{i \in\{0, m, \ldots, M\}} \pi\left(v^{i} \mid X^{i}, \Theta\right)
$$

where we define $\hat{\mathrm{Y}}_{M}=\left(Y^{0}, Y^{1}, \ldots, Y^{M-1}\right)$ and is simply the vector of latent values up to time $t_{M}$. Hence our target is

$$
\pi_{M}\left(\Theta, Y^{M}\right) \propto \pi_{j}\left(\Theta, Y^{j}\right) \pi\left(v^{M} \mid X^{M}, \Theta\right) \prod_{i=j}^{M-1} \pi\left(Y^{i+1} \mid Y^{i}, \Theta\right)
$$

with $Y^{j}, \ldots, Y^{M-1}$ integrated out. As $\pi_{j}\left(\Theta, Y^{j}\right)$ has no analytic form, we recursively approximate $\Theta, Y^{j} \mid D_{j}$ by the "particles" $\left\{\left(\Theta_{(s)}, Y_{(s)}^{j}\right), s=1, \ldots, S\right\}$ with each $\Theta_{(s)}, Y_{(s)}^{j}$ having a discrete probablity mass of $w_{(s)}^{j}=1 / S$. We assume that as $S \rightarrow \infty$, the particles approximate the filtering density, $\pi_{j}\left(\Theta, Y^{j}\right)$ increasingly well. Filters which treat the discrete support generated by the particles as the true (filtering) distribution are known as particle filters. Various implementations of particle filters have been proposed in the literature including sampling/importance resampling (Doucet et al., 2000) and MCMC (Pitt and Shephard, 1999). Here we focus on an MCMC approach.

In the first step of our MCMC scheme, propose $\left(\Theta_{*}, Y_{*}^{j}\right)$ from $\pi_{j}\left(\Theta, Y^{j}\right)$ using the kernel density estimate of $\pi_{j}(\cdot, \cdot)$. First select an integer, $u$, uniformly from the set $\{1, \ldots, S\}$ and then put

$$
\left(\Theta_{*}, Y_{*}^{j}\right)^{\prime} \sim \mathrm{N}\left\{\left(\Theta_{(u)}, Y_{(u)}^{j}\right)^{\prime}, \omega^{2} B\right\}
$$

where $B$ is the Monte Carlo posterior variance and the overall scale of the kernel is a function of the smoothing parameter $\omega^{2}$, usually around 0.02 . Note that the kernel density estimate is necessary to avoid sample impoverishment which occurs when the number of distinct particles become arbitrarily small (Golightly and Wilkinson, 2005b). For large datasets however, Liu and West (2001) suggest that the random disturbances add up to give "information loss" over time (as the kernel density function is always over-dispersed relative to the posterior sample by a factor $1+\omega^{2}$ ). A kernel shrinkage method can be used to correct this (see Liu and West (2001) for a discussion).

We then draw $Y_{*}^{i+1}$ for $i=j, \ldots, M-1$ recursively from $\tilde{\pi}\left(Y_{*}^{i+1} \mid Y_{*}^{i}, v^{M}, \Theta_{*}\right)$ and accept the move with probabilty $\min \{1, \alpha\}$ where

$$
\alpha=\frac{\left[\pi\left(v^{M} \mid X_{*}^{M}, \Theta_{*}\right) \prod_{i=j}^{M-1} \pi\left(Y_{*}^{i+1} \mid Y_{*}^{i}, \Theta_{*}\right)\right]\left[\prod_{i=j}^{M-1} \tilde{\pi}\left(Y^{i+1} \mid Y^{i}, v^{M}, \Theta\right)\right]}{\left[\pi\left(v^{M} \mid X^{M}, \Theta\right) \prod_{i=j}^{M-1} \pi\left(Y^{i+1} \mid Y^{i}, \Theta\right)\right]\left[\prod_{i=j}^{M-1} \tilde{\pi}\left(Y_{*}^{i+1} \mid Y_{*}^{i}, v^{M}, \Theta_{*}\right)\right]} .
$$


We repeat this step for $S$ iterations (after a burn-in period) and discard all draws except $\Theta$ and $Y^{M}$ to obtain the desired sample, $\left\{\left(\Theta_{(s)}, Y_{(s)}^{M}\right), s=\right.$ $1, \ldots, S\}$ from $\pi_{M}\left(\Theta, Y^{M}\right)$. Note that variations on the sampling strategy are possible; in Golightly and Wilkinson (2006), $X_{*}^{M}$ is drawn from $\pi\left(X_{*}^{M} \mid v^{M}, \Theta_{*}\right)$ and then $Y_{*}^{j+1}, \ldots, Y_{*}^{M-1}$ are simulated using the modified bridge density, (A.5), further conditioned on $X_{*}^{M}$. Finally $Z_{*}^{M}$ is drawn from the Euler transition density conditioned on $X_{*}^{M}, \pi\left(Z_{*}^{M} \mid Y_{*}^{M-1}, X_{*}^{M}, \Theta_{*}\right)$.

\section{Simulation Study: Auto-regulatory Gene Network}

Transcriptional regulation has been studied extensively in both prokaryotic and eukaryotic organisms (see, for example McAdams and Arkin (1999) and Latchman (2002)). In a simple model of prokaryotic auto regulation, a protein (I) coded for by a gene (i) represses its own transcription and also the transcription of another gene, (g) by binding to a regulatory region upstream of the gene. We simplify the repression mechanisms with the reactions,

$$
\begin{aligned}
& R_{1}: \mathrm{I}+\mathrm{i} \longrightarrow \mathrm{I} \cdot \mathrm{i} \\
& R_{2}: \mathrm{I} \cdot \mathrm{i} \longrightarrow \mathrm{I}+\mathrm{i} \\
& R_{3}: \mathrm{I}+\mathrm{g} \longrightarrow \mathrm{I} \cdot \mathrm{g} \\
& R_{4}: \mathrm{I} \cdot \mathrm{g} \longrightarrow \mathrm{I}+\mathrm{g}
\end{aligned}
$$

We represent the transcription of $i$, the binding of a ribosome to mRNA, the translation of mRNA and the folding of the resulting polypetide chain into a folding protein, I, by

$$
R_{5}: \mathrm{i} \longrightarrow \mathrm{i}+\mathrm{r}_{\mathrm{i}}, R_{6}: \mathrm{r}_{\mathrm{i}} \longrightarrow \mathrm{r}_{\mathrm{i}}+\mathrm{I}
$$

Similarly, we represent the transcription of $\mathrm{g}$ and translation mechanism by

$$
R_{7}: \mathrm{g} \longrightarrow \mathrm{g}+\mathrm{r}_{\mathrm{g}}, R_{8}: \mathrm{r}_{\mathrm{g}} \longrightarrow \mathrm{r}_{\mathrm{g}}+\mathrm{G}
$$

Finally, the model is completed by mRNA degradation,

$$
R_{9}: \mathrm{r}_{\mathrm{i}} \longrightarrow \emptyset, R_{10}: \mathrm{r}_{\mathrm{g}} \longrightarrow \emptyset
$$

and protein degradation,

$$
R_{11}: \mathrm{I} \longrightarrow \emptyset, R_{12}: \mathrm{G} \longrightarrow \emptyset \text {. }
$$

For a detailed discussion of gene regulation see Ptashne (1992) and Latchman (2002). 
We illustrate the methodology of Section 3 by estimating the rate constants governing the diffusion approximation of the network given by the above reactions. We start by assuming a rate constant, $c_{i}$, for each reaction, $R_{i}$ and consider the time evolution of the system as a Markov process with state

$$
Y=\left(Y_{1}, Y_{2}, \ldots, Y_{8}\right)^{\prime}=\left(\mathrm{I}, \mathrm{G}, \mathrm{I} \cdot \mathrm{i}, \mathrm{I} \cdot \mathrm{g}, \mathrm{i}, \mathrm{g}, \mathrm{r}_{\mathrm{i}}, \mathrm{r}_{\mathrm{g}}\right)^{\prime}
$$

denoting the number of molecules of each respective species at time $t$. With the species ordered by the preceding equation, we can represent our network by the $12 \times 8$ net effect reaction matix $A=\left(a_{i j}\right)$ where $a_{i j}$ denotes the net gain in the number of molecules of species $j$ after reaction $R_{i}$ has occurred. We obtain

$$
\mathrm{A}^{\prime}=\left(\begin{array}{cccccccccccc}
-1 & 1 & -1 & 1 & 0 & 1 & 0 & 0 & 0 & 0 & -1 & 0 \\
0 & 0 & 0 & 0 & 0 & 0 & 0 & 1 & 0 & 0 & 0 & -1 \\
1 & -1 & 0 & 0 & 0 & 0 & 0 & 0 & 0 & 0 & 0 & 0 \\
0 & 0 & 1 & -1 & 0 & 0 & 0 & 0 & 0 & 0 & 0 & 0 \\
-1 & 1 & 0 & 0 & 0 & 0 & 0 & 0 & 0 & 0 & 0 & 0 \\
0 & 0 & -1 & 1 & 0 & 0 & 0 & 0 & 0 & 0 & 0 & 0 \\
0 & 0 & 0 & 0 & 1 & 0 & 0 & 0 & -1 & 0 & 0 & 0 \\
0 & 0 & 0 & 0 & 0 & 0 & 1 & 0 & 0 & -1 & 0 & 0
\end{array}\right)
$$

We now calculate the diffusion approximation by applying the Fokker-Planck equation to the chemical master equation associated with the process. See Golightly and Wilkinson (2005a) and Wilkinson (2006) for further details. It is easily seen that drift and diffusion coefficients are given by

$$
\mu(Y, \Theta)=\mathrm{A}^{\prime} h(Y, \Theta), \beta(Y, \Theta)=\mathrm{A}^{\prime} \operatorname{diag}\{h(Y, \Theta)\} \mathrm{A},
$$

where $h(Y, \Theta)$ is the column vector of hazards $h_{i}\left(Y, c_{i}\right)$. Before application of the above equation, it should be noted that the net effect matrix $\mathrm{A}$ is not of full rank (as the number of molecules of $\mathrm{I} \cdot \mathrm{i}$ and $\mathrm{I} \cdot \mathrm{g}$ are related to the number of molecules of $\mathrm{i}$ and $\mathrm{g}$ respectively) and this will yield a rank degenerate diffusion matrix. Inspection of (24) reveals that adding row 3 of $\mathrm{A}^{\prime}$ to row 5 implies

$$
\mathrm{I} \cdot \mathrm{i}+\mathrm{i}=K_{1}
$$

and similarly, adding row 4 to row 6 yields

$$
\mathrm{l} \cdot \mathrm{g}+\mathrm{g}=K_{2}
$$

where $K_{1}$ and $K_{2}$ are known as conservation constants. As this rank degeneracy will complicate the inference methods of Sections 3 and 4, we remove 
rows 3 and 4 from $\mathrm{A}^{\prime}$ to obtain $\mathrm{A}$ of full rank. We then use (26) and (27) to substitute $K_{1}-\mathrm{i}$ and $K_{2}-\mathrm{g}$ for $\mathrm{I} \cdot \mathrm{i}$ and $\mathrm{I} \cdot \mathrm{g}$ respectively to reduce our model to one involving just 6 chemical species, $Y=\left(\mathrm{I}, \mathrm{G}, \mathrm{i}, \mathrm{g}, \mathrm{r}_{\mathrm{i}}, \mathrm{r}_{\mathrm{g}}\right)^{\prime}$. The full diffusion approximation can then be computed using (25), for example,

$$
\mu(Y, \Theta)=\left(\begin{array}{c}
c_{2}\left(K_{1}-\mathrm{i}\right)+c_{4}\left(K_{2}-\mathrm{g}\right)+c_{6} \mathrm{r}_{\mathrm{i}}-c_{1} \mathrm{li}-c_{3} \mid \mathrm{g}-c_{11} \mathrm{l} \\
c_{8} \mathrm{r}_{\mathrm{g}}-c_{12} \mathrm{G} \\
c_{2}\left(K_{1}-\mathrm{i}\right)-c_{1} \mathrm{li} \\
c_{4}\left(K_{2}-\mathrm{g}\right)-c_{3} \mathrm{lg} \\
c_{5} \mathrm{i}-c_{9} \mathrm{r}_{\mathrm{i}} \\
c_{7} \mathrm{~g}-c_{10} \mathrm{r}_{\mathrm{g}}
\end{array}\right) .
$$

Though we work with the reduced system in Sections 5.1 and 5.2, both sampling schemes can be implemented for the full model by working with the Moore-Penrose generalised inverse of the diffusion matrix.

\subsection{Results: Fully Observed Model}

We repeat the sampling experiments of Golightly and Wilkinson (2006) by implementing the innovation scheme and the simulation filter for the fully observed case. We assume that we observe $Y(t)=\left(\mathrm{I}(t), \mathrm{G}(t), \mathrm{i}(t), \mathrm{g}(t), \mathrm{r}_{\mathrm{i}}(t), \mathrm{r}_{\mathrm{g}}(t)\right)^{\prime}$ at all times $t$ and consider data, $\mathcal{D}_{1}$, consisting of 30 observations on [0,29] with each data point subject to Gaussian error with zero mean and variance $\sigma^{2}=3$ (so that $\Sigma=\sigma^{2}$ I in (2)), which we assume to be unknown. Note that all data (including the datsets considered in Section 5.2) were simulated from the true discrete stochastic kinetic model using the Gillespie algorithm, and not from the diffusion approximation. Values for $\left(c_{1}, \ldots, c_{12}\right)$ that produced the data are $0.08,0.82,0.09,0.9,0.25,0.1,0.35,0.3,0.1,0.1,0.12,0.1$ and we place Uniform $\mathrm{U}(-5,1)$ priors on each $\log \left(c_{i}\right)$, for $i=1, \ldots, 12$ and $\sigma$. Note that $K_{1}$ and $K_{2}$ (the number of copies of each gene) are set to be 10 and are assumed to be known.

We run the innovation scheme and the simulation filter for each $m=2,8,20$; the innovation scheme is run for 4 million iterations with a thin of 200 , yielding a sample of 20,000 to be used as the main monitoring run. We run the simulation filter for 4.5 million iterations with a thin of 150 , giving $S=30,000$ particles at each time point. Posterior means and standard deviations for $\Theta$ based on the output of the innovation scheme and the simulation filter are reported in Tables A.1 and A.2 respectively. Fig. A.1 gives trace and posterior density plots obtained from the output of each sampler and $m=2$. Autocorrelation plots for a selection of parameters can be found in Fig. A.2. 
[Figure 1 about here.]

[Figure 2 about here.]

[Table 1 about here.]

[Table 2 about here.]

Tables A.1 and A.2 reveal that both sampling strategies produce estimates that are close to the true values that produced the sample data. Inspection of Fig. A.1 reveals that both the innovation scheme and the simulation filter produce estimates that are consistent with one another. The advantage of including latent variables in the estimation is abundently clear; for example, running the innovation scheme with $m=2(\Delta t=0.5)$ yields posterior means of $c_{8}$ and $c_{12}$ as 0.212 and 0.074 whilst true values are 0.3 and 0.1 respectively. Increasing $m$ to $20(\Delta t=0.05)$ gives a marked increase in accuracy with posterior means of 0.241 and 0.086. Note that whilst posterior means for $c_{1}, c_{2}$, $c_{3}$ and $c_{4}$ are fairly inaccurate for all choices of $m$, the posterior means of $c_{1} / c_{2}$ and $c_{3} / c_{4}$, corresponding to the overall propensities for the reversible reactions, $R_{1}$ and $R_{2}, R_{3}$ and $R_{4}$, are very close to the true values that produced the data.

We assess the relative performance of each scheme by studying the sample autocorrelation functions for each parameter; Fig. A.2 shows that as we increase $m$, autocorrelations still die down very quickly for both schemes. In fact, at lag 100, parameter draws are no more autocorrelated for $m=20$ than for $m=2$.

\subsection{Results: Partially Observed Model}

We now turn out attention to the application of the innovation scheme to the partially observed model. Again, we repeat the experiments of Golightly and Wilkinson (2006) by considering 3 datasets; $\mathcal{D}_{2}, \mathcal{D}_{3}$ and $\mathcal{D}_{4}$, each independently simulated with stochastic rate constants, $c_{1}, \ldots, c_{12}$ as in Section 5.1. $\mathcal{D}_{2}$ consists of 30 observations on $X(t)=\left(\mathrm{I}(t), \mathrm{G}(t), \mathrm{r}_{\mathrm{i}}(t), \mathrm{r}_{\mathrm{g}}(t)\right)^{\prime}$ with each data point subject to measurement error with variance $\sigma^{2}=3.0$. $\mathcal{D}_{3}$ contains 40 observations on protein levels only; $X(t)=(\mathrm{I}(t), \mathrm{G}(t))^{\prime}$ and the variance of the measurement error is $\sigma^{2}=3.0$. Finally, $\mathcal{D}_{4}$ contains 20 observations on RNA levels; $X(t)=\left(r_{\mathrm{i}}(t), \mathrm{r}_{\mathrm{g}}(t)\right)^{\prime}$ with $\sigma^{2}=2.0$. For each dataset, we assume that the variance of the measurement error is known and that the number of copies of each gene is known to be $K_{1}=K_{2}=10$. As in Section 5.1, we place uniform priors on each $\log \left(c_{i}\right)$ and also on $\log \left(Z^{0}\right)$. We run the innovation scheme and the simulation filter for each dataset with $m=20 ; 4$ million iterations with a thin of 200 are performed, leaving 20,000 iterations for the 
main monitoring run. Figs. A.3-A.4 and Tables A.3 and A.4 summarise the posterior distribution based on the MCMC output of each scheme.

[Figure 3 about here.]

[Figure 4 about here.]

[Table 3 about here.]

[Table 4 about here.]

Inspection of Fig. A.3 reveals that both samplers produce estimates that are close to the true values that generated the sample data and running scheme until convergence gives estimates that are consistent with each other. As shown in Fig. A.4, autocorrelations obtained from each scheme die down very quickly, though the simulation filter yields sample values which are generally much less autocorrelated than those of the innovation scheme.

As found in Golightly and Wilkinson (2006), when observing a subset of species, estimates are in general more accurate for rate constants governing reactions involving those species. For example $c_{11}$ and $c_{12}$ (pertaining to protein degradation reactions given by (23) with true values of 0.12 and 0.1 are estimated to be 0.126 and 0.157 using the innovation scheme with 20 observations on RNA levels $\left(\mathcal{D}_{4}\right)$. However, when using 40 observations on protein levels $\left(\mathcal{D}_{3}\right)$, we see an increase in accuracy with estimates of 0.108 and 0.086 respectively.

Note that both the innovation scheme and simulation filter are coded in $\mathrm{C}$ and executed on a Pentium IV $1.8 \mathrm{GHz}$ processor. Computational times for the innovation scheme and simulation filter for a datset of length 20 (simulated from the model), with $m=20$ and 20,000 iterations are approximately 4 and 20 minutes respectively.

\section{Discussion}

By considering the analysis of a partially and discretely observed SDE as a missing data problem, Bayesian inference is usually undertaken by adopting a Gibbs sampler or MCMC approach. Whilst naive schemes (such as those of Eraker (2001) and Golightly and Wilkinson (2005a)) can be satisfactory for small amounts of augmentation $(m \leq 5)$, they can break down as $m$ increases due to high dependence between the latent data and parameters governing the volatility coefficient (Roberts and Stramer, 2001). In this paper, by employing a reparameterisation similar to that of Chib et al. (2004), we have provided an MCMC scheme to sample diffusion parameters, that is not afflicted by this 
dependence issue. By applying the methodology to the estimation of parameters governing the diffusion approximation of a regulatory gene network, we have shown that the scheme works well for high dimensional diffusions that are observed with error.

The sequential simulation filter of Golightly and Wilkinson (2005b) and Golightly and Wilkinson (2006) was also considered. Whilst the simulation filter does not break down for large $m$, posterior samples do ultimately degenerate when large datasets are used. Although this is not a significant practical problem in the context of stochastic biochemical rate constant estimation (where datasets are typically relatively small), it does limit the utility of the algorithm. The innovation scheme, on the other hand, does not suffer from this problem. 


\section{A Calculating the Modified Diffusion Bridge}

\section{A.1 Fixed end points}

Consider times $t_{j}$ and $t_{M}$ (where $j$ is an integer multiple of $m$ and $M=j+m$ ) such that the corresponding states are $Y^{j}$ and $Y^{M}$ which we will assume to be fixed. The modified diffusion bridge is constructed by sampling $Y^{i+1}$ for $i=0, \ldots, M-2$ from a Gaussian approximation to $\pi\left(Y^{i+1} \mid Y^{i}, Y^{M}\right)$ for which we denote the approximate density by $\tilde{\pi}\left(Y^{i+1} \mid Y^{i}, Y^{M}\right)$. Note that we suppress the dependence of this density on $\Theta$ for simplicity.

We derive $\tilde{\pi}\left(Y^{i+1} \mid Y^{i}, Y^{M}\right)$ by formulating the approximate joint density of $Y^{i+1}$ and $Y^{M}$ (conditional on $Y^{i}$ ) and using MVN theory to condition on $Y^{M}$. We therefore start with the density of $Y^{M}$ conditional on $Y^{i+1}$ which we obtain using a very crude Euler approximation,

$$
\tilde{\pi}\left(Y^{M} \mid Y^{i+1}\right)=\phi\left(Y^{M} ; Y^{i+1}+\mu_{i+1} \Delta^{+}, \beta_{i+1} \Delta^{+}\right)
$$

where $\Delta^{+}=(M-i-1) \Delta t$ and we use the short-hand notation of Chapter 3 , writing $\mu_{i+1}$ and $\beta_{i+1}$ for $\mu\left(Y^{i+1}\right)$ and $\beta\left(Y^{i+1}\right)$. To give a linear Gaussian structure, we approximate (A.1) further by noting that $\mu$ and $\beta$ are locally constant (by assumption). Estimating $\mu_{i+1}$ and $\beta_{i+1}$ by $\mu_{i}$ and $\beta_{i}$ respectively, we obtain

$$
\tilde{\pi}\left(Y^{M} \mid Y^{i+1}\right)=\phi\left(Y^{M} ; Y^{i+1}+\mu_{i} \Delta^{+}, \beta_{i} \Delta^{+}\right) .
$$

The density $\pi\left(Y^{i+1} \mid Y^{i}\right)$ is the one step ahead Euler transition density given by

$$
\pi\left(Y^{i+1} \mid Y^{i}\right)=\phi\left(Y^{i+1} ; Y^{i}+\mu_{i} \Delta t, \beta_{i} \Delta t\right)
$$

and we can therefore construct the approximate joint density of $Y^{i+1}$ and $Y^{M}$ (conditional on $Y^{i}$ ) using MVN conditioning results which yield

$$
\left(\begin{array}{c}
Y^{i+1} \\
Y^{M}
\end{array}\right) \sim \mathrm{N}_{2 d}\left\{\left(\begin{array}{c}
Y^{i}+\mu_{i} \Delta t \\
Y^{i}+\mu_{i} \Delta^{-}
\end{array}\right),\left(\begin{array}{c}
\beta_{i} \Delta t \beta_{i} \Delta t \\
\beta_{i} \Delta t \beta_{i} \Delta^{-}
\end{array}\right)\right\}
$$

where $\Delta^{-}=(M-i) \Delta t$. We now condition (A.4) on $Y^{M}$ to give

$$
\tilde{\pi}\left(Y^{i+1} \mid Y^{i}, Y^{M}\right)=\phi\left(Y^{i+1} ; Y^{i}+\frac{Y^{M}-Y^{i}}{M-i}, \frac{M-i-1}{M-i} \beta_{i} \Delta t\right) .
$$

This is the form of the modified bridge given in Durham and Gallant (2002). We now consider two extensions of the diffusion bridge construct. 


\section{A.2 Conditioning on $v^{M}$}

It may be the case that we only have one fixed end-point, $Y^{j}$ and we wish to draw $Y^{j+1}, \ldots, Y^{M}$ conditional on $Y^{j}$ and the (noisy) observation $v^{M}$. We achieve this by sampling $Y^{i+1}$ for $i=j, \ldots, M-1$ from a Gaussian approximation to $\pi\left(Y^{i+1} \mid Y^{i}, v^{M}\right)$ which we denote by $\tilde{\pi}\left(Y^{i+1} \mid Y^{i}, v^{M}\right)$. We start by approximating the density of $v^{M}$ conditional on $Y^{i+1}$ as

$$
\tilde{\pi}\left(v^{M} \mid Y^{i+1}\right)=\phi\left(v^{M} ; X^{i+1}+\mu_{i}^{x x} \Delta^{+}, \beta_{i}^{x x} \Delta^{+}\right)
$$

using a partition of $\mu_{i}$ and $\beta_{i}$ of the form

$$
\mu_{i}=\left(\begin{array}{c}
\mu_{i}^{x} \\
\mu_{i}^{z}
\end{array}\right), \beta_{i}=\left(\begin{array}{cc}
\beta_{i}^{x x} & \beta_{i}^{x z} \\
\beta_{i}^{z x} & \beta_{i}^{z z}
\end{array}\right)
$$

Recall also that $\Sigma$ is the variance of the measurement error density, (6). Combining (A.6) with the one step ahead Euler transition, (A.3), we obtain the approximate joint density of $Y^{i+1}$ and $v^{M}$ (conditional on $Y^{i}$ ) as

$$
\left(\begin{array}{c}
Y^{i+1} \\
v^{M}
\end{array}\right) \sim \mathrm{N}_{d+d_{1}}\left\{\left(\begin{array}{c}
Y^{i}+\mu_{i} \Delta t \\
X^{i}+\mu_{i}^{x} \Delta^{-}
\end{array}\right),\left(\begin{array}{cc}
\beta_{i} \Delta t & C_{i} \Delta t \\
C_{i}^{\prime} \Delta t & \beta_{i}^{x x} \Delta^{-}+\Sigma
\end{array}\right)\right\},
$$

where $C_{i}^{\prime}=\left(\beta_{i}^{x x}, \beta_{i}^{x z}\right)$. Conditioning (A.7) on $v^{M}$ yields

$$
\tilde{\pi}\left(Y^{i+1} \mid Y^{i}, v^{M}\right)=\phi\left(Y^{i+1} ; Y^{i}+\psi_{i} \Delta t, \gamma_{i} \Delta t\right)
$$

where

$$
\psi_{i}=\mu_{i}+C_{i}\left(\beta_{i}^{x x} \Delta^{-}+\Sigma\right)^{-1}\left(v^{M}-\left[X^{i}+\mu_{i}^{x x} \Delta^{-}\right]\right)
$$

and

$$
\gamma_{i}=\beta_{i}-C_{i}\left(\beta_{i}^{x x} \Delta^{-}+\Sigma\right)^{-1} C_{i}^{\prime} \Delta t
$$

\section{A.3 Fixed end points with mid-point conditioning}

Consider the block updating scheme of Section 3.1 and the task of sampling $Y^{j+1}, \ldots, Y^{M}$ conditional on the end points $Y^{j}, Y^{M^{+}}$and also the noisy midpoint, $v^{M}$ (recall that $j$ is a multiple of $m, M=j+m$ and $M^{+}=M+m$ ). This is achieved by sampling $Y^{i+1}$ for $i=j, \ldots, M-1$ from a Gaussian approximation to $\pi\left(Y^{i+1} \mid Y^{i}, v^{M}, Y^{M^{+}}\right)$which we denote by $\tilde{\pi}\left(Y^{i+1} \mid Y^{i}, v^{M}, Y^{M^{+}}\right)$. We start by approximating the density of $Y^{M^{+}}$conditional on $Y^{i+1}$ (and $v^{M}$ ) as

$$
\tilde{\pi}\left(Y^{M^{+}} \mid Y^{i+1}\right)=\phi\left(Y^{M^{+}} ; Y^{i+1}+\mu_{i} \Delta^{++}, \beta_{i} \Delta^{++}\right),
$$


where $\Delta^{++}=\left(M^{+}-j-1\right) \Delta t$. This ensures that we have a linear Gaussian system. Then, combining (A.11) with approximate joint density of $Y^{i+1}$ and $v^{M}$, (A.7), we obtain the approximate joint density of $Y^{i+1}$ and $Y^{M^{+}}$and $v^{M}$ (conditional on $Y^{i}$ ) as

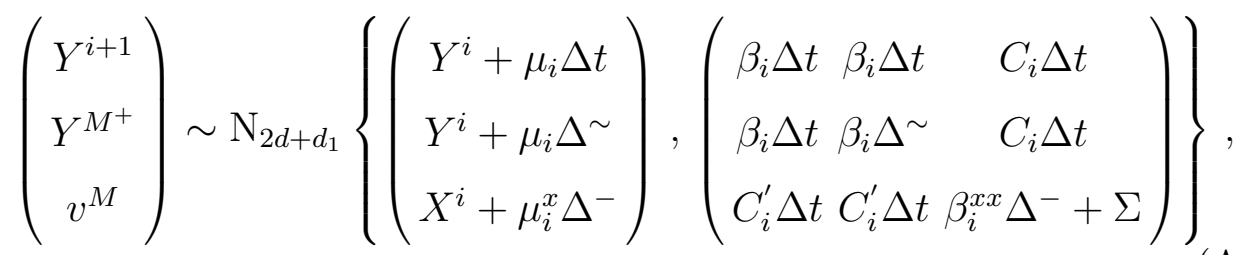

where $\Delta^{\sim}=\left(M^{+}-i\right) \Delta t$. Conditioning (A.12) on $v^{M}$ and $Y^{M^{+}}$yields

$$
\tilde{\pi}\left(Y^{i+1} \mid Y^{i}, v^{M}\right)=\phi\left(Y^{i+1} ; Y^{i}+\psi_{i}^{\prime} \Delta t, \gamma_{i}^{\prime} \Delta t\right)
$$

where

$$
\psi_{i}^{\prime}=\mu_{i}+\left(\beta_{i}, C_{i}\right)\left(\begin{array}{cc}
\beta_{i} \Delta^{\sim} & C_{i} \Delta t \\
C_{i}^{\prime} \Delta t & \beta_{i}^{x x} \Delta^{-}+\Sigma
\end{array}\right)^{-1}\left(\begin{array}{c}
Y^{M^{+}}-\left[Y^{i}+\mu_{i}^{x} \Delta^{\sim}\right] \\
v^{M}-\left[X^{i}+\mu_{i}^{x} \Delta^{-}\right]
\end{array}\right)
$$

and

$$
\gamma_{i}^{\prime}=\beta_{i}-\left(\beta_{i}, C_{i}\right)\left(\begin{array}{cc}
\beta_{i} \Delta^{\sim} & C_{i} \Delta t \\
C_{i}^{\prime} \Delta t & \beta_{i}^{x x} \Delta^{-}+\Sigma
\end{array}\right)^{-1}\left(\begin{array}{l}
\beta_{i} \Delta t \\
C_{i}^{\prime} \Delta t
\end{array}\right)
$$

\section{References}

Arkin, A., Ross, J., McAdams, H. H., 1998. Stochastic kinetic analysis of developmental pathway bifurcation in phage lambda-infected Escherichia coli cells. Genetics (149), 633-648.

Berzuini, C., Best, N. G., Gilks, W. R., Larizza, C., 1997. Dynamic conditional independence models and markov chain monte carlo methods. Journal of the American Statistical Association 92 (440), 1403-1412.

Beskos, A., Papaspiliopoulos, O., Roberts, G. O., Fearnhead, P., 2006. Exact and computationally efficient likelihood-based estimation for discretely observed diffusion processes. Journal of the Royal Statistical Society, Series B: Statistical Methodology 68, 1-29.

Bibby, B. M., Sørensen, M., 1995. Martingale estimating functions for discretely observed diffusion processes. Bernouilli 1, 17-39.

Black, F., Scholes, M. S., 1973. The pricing of options and corporate liabilities. Journal of Political Economy 81, 637-659.

Chan, K. C., Karolyi, G. A., Longstaff, F. A., Sanders, A. B., 1992. An empirical comparison of alternative models of the short-term interest. Journal of Finance 47, 1209-1228. 
Chib, S., Pitt, M. K., Shephard, N., 2004. Likelihood based inference for diffusion driven models. In submission.

Cox, J. C., Ingersoll, J. E., Ross, S. A., 1985. A theory of the term structure of interest rates. Econometrica 53, 385-407.

Del Moral, P., Jacod, J., Protter, P., 2002. The Monte Carlo method for filtering with discrete-time observations. Probability Theory and Related Fields 120, 346-368.

Doucet, A., Godsill, S., Andrieu, C., 2000. On sequential Monte Carlo sampling methods for Bayesian filtering. Statistics and Computing 10, 197-208.

Durham, G. B., Gallant, R. A., 2002. Numerical techniques for maximum likelihood estimation of continuous-time diffusion processes. Journal of Business and Economic Statistics 20, 279-316.

Elerian, O., Chib, S., Shephard, N., 2001. Likelihood inference for discretely observed nonlinear diffusions. Econometrica 69 (4), 959-993.

Eraker, B., 2001. MCMC analysis of diffusion models with application to finance. Journal of Business and Economic Statistics 19, 177-191.

Gillespie, D. T., 1992. A rigorous derivation of the chemical master equation. Physica A 188, 404-425.

Golightly, A., Wilkinson, D. J., 2005a. Bayesian inference for stochastic kinetic models using a diffusion approximation. Biometrics 61 (3), 781-788.

Golightly, A., Wilkinson, D. J., 2005b. Bayesian sequential inference for nonlinear multivariate diffusions. In Submission.

Golightly, A., Wilkinson, D. J., 2006. Bayesian sequential inference for stochastic kinetic biochemical network models. Journal of Computational Biology 13 (3), 838-851.

Johannes, M. S., Polson, N. G., Stroud, J. R., 2006. Optimal filtering of jump diffusions: Extracting latent states from asset prices.

Latchman, D., 2002. Gene Regulation: A Eukaryotic Perspective. BIOS Scientific Publishers.

Liu, J., West, M., 2001. Combined parameter and state estimation in simulation-based filtering. In: Doucet, A., de Freitas, N., Gordon, N. (Eds.), Sequential Monte Carlo Methods in Practice.

McAdams, H. H., Arkin, A., 1999. Its a noisy business: Genetic regulation at the nanomolar scale. Trends in Genetics 15, 65-69.

Øksendal, B., 1995. Stochastic Differential Equations: An Introduction with Applications, 6th Edition. Springer-Verlag.

Papaspiliopolous, O., Roberts, G. O., Skôld, M., 2003. Non-centered parameterisations for hierarchical models and data augmentation. In: Bernardo, J. M., Bayarri, M. J., Berger, J. O., Dawid, A. P., Heckerman, D., Smith, A. F. M., West, M. (Eds.), Bayesian Statistics 7. Oxford University Press, pp. 307-326.

Pedersen, A., 1995. A new approach to maximum likelihood estimation for stochastic differential equations based on discrete observations. Scandinavian Journal of Statistics 1995 (22), 55-71.

Pitt, M. K., Shephard, N., 1999. Filtering via simulation: Auxiliary particle 
filters. Journal of the American Statistical Association 446 (94), 590-599.

Ptashne, M., 1992. A Genetic Switch: Phage $\lambda$ and Higher Organisms, 2nd Edition. Cell Press and Blackwell Scientific Publications.

Roberts, G. O., Stramer, O., 2001. On inference for partially observed nonlinear diffusion models using the Metropolis-Hastings algorithm. Biometrika 88 (4), 603-621.

Shephard, N., Pitt, M. K., 1997. Likelihood analysis of non-Gaussian measurement time series. Biometrika 84, 653-667.

Stroud, J. R., Polson, N. G., Muller, P., 2004. Practical filtering for stochastic volatility models. In: Harvey, A., Koopman, S. J., Shephard, N. (Eds.), State Space and Unobserved Components Models. Cambridge Press, pp. 236-247.

Tanner, M. A., Wong, W. H., 1987. The calculation of posterior distributions by data augmentation. Journal of the American Statistical Association 82 (398), 528-540.

Wilkinson, D. J., 2003. Disscussion to 'Non centred parameterisations for hierarchical models and data augmentation' by Papaspiliopoulos, Roberts and Skold. In: Bayesian Statistics 7. Oxford Science Publications, pp. 323-324.

Wilkinson, D. J., 2006. Stochastic Modelling for Systems Biology. Chapman \& Hall / CRC Press. 


\section{List of Figures}

A.1 Trace plots and posterior density plots obtained for $c_{5}, c_{6}, c_{8}$, $c_{9}, c_{10}$ and $c_{12}$ from the output of (a) the innovation scheme (solid line in (c)) and (b) the simulation filter (dashed line in (c)), using dataset $\mathcal{D}_{1}$ and $m=2$.

A.2 Autocorrelation plots for $c_{5}, c_{6}, c_{8}, c_{9}, c_{10}$ and $c_{12}$ from the output of the innovation scheme (solid line) and the simulation filter (dashed line) using dataset $\mathcal{D}_{1}$ and (a) $m=2$, (b) $m=8$ and (c) $m=20$.

A.3 Posterior density plots for $c_{2}, c_{3}, c_{7}, c_{9}, c_{10}$ and $c_{12}$ from the output of the innovation scheme (solid line) and the simulation filter (dashed line) using datasets (a) $\mathcal{D}_{2}$, (b) $\mathcal{D}_{3}$ and (c) $\mathcal{D}_{4}$.

A.4 Autocorrelation plots for $c_{2}, c_{3}, c_{7}, c_{9}, c_{10}$ and $c_{12}$ from the output of the innovation scheme (solid line) and the simulation filter (dashed line) using datasets (a) $\mathcal{D}_{2}$, (b) $\mathcal{D}_{3}$ and (c) $\mathcal{D}_{4}$. 
(a)
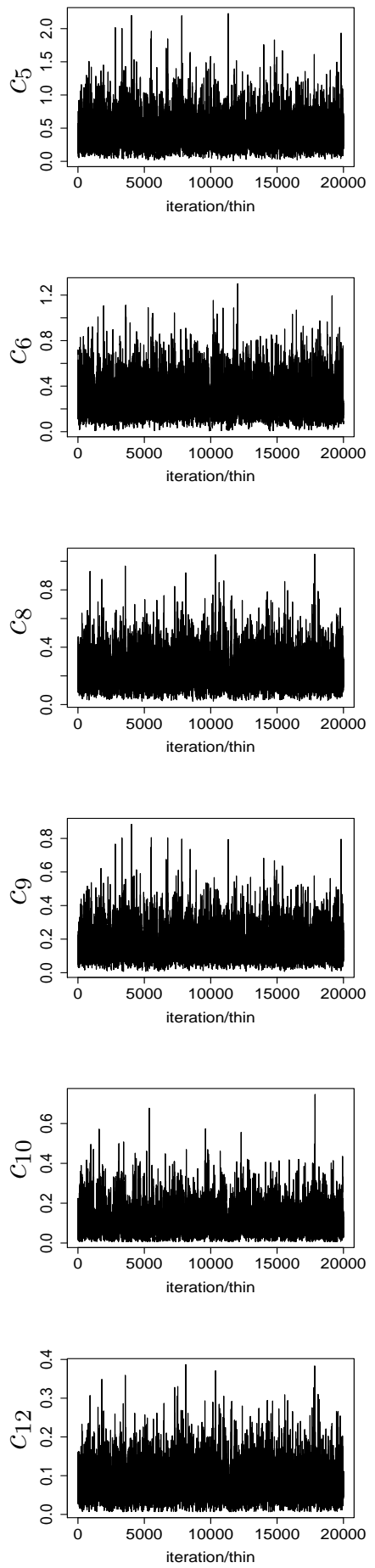

(b)
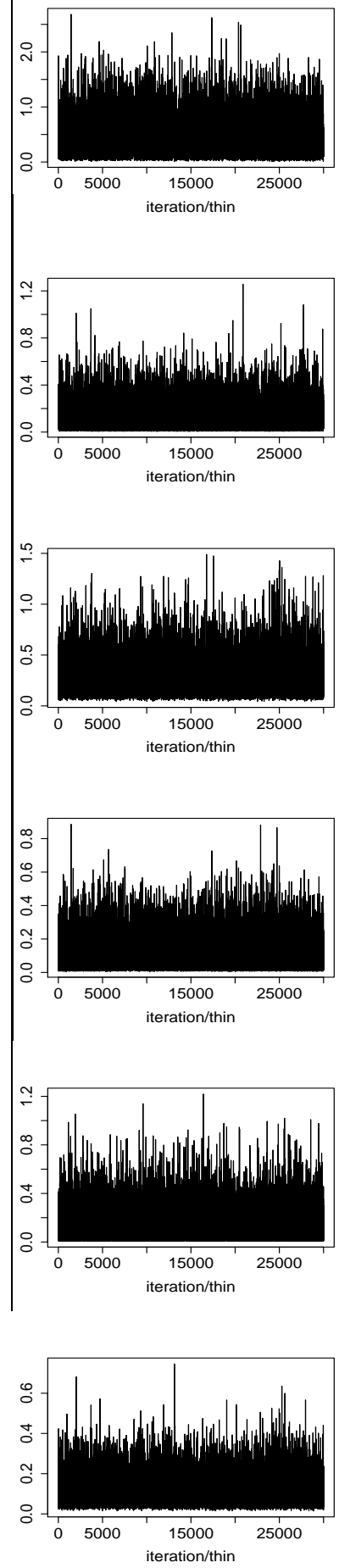
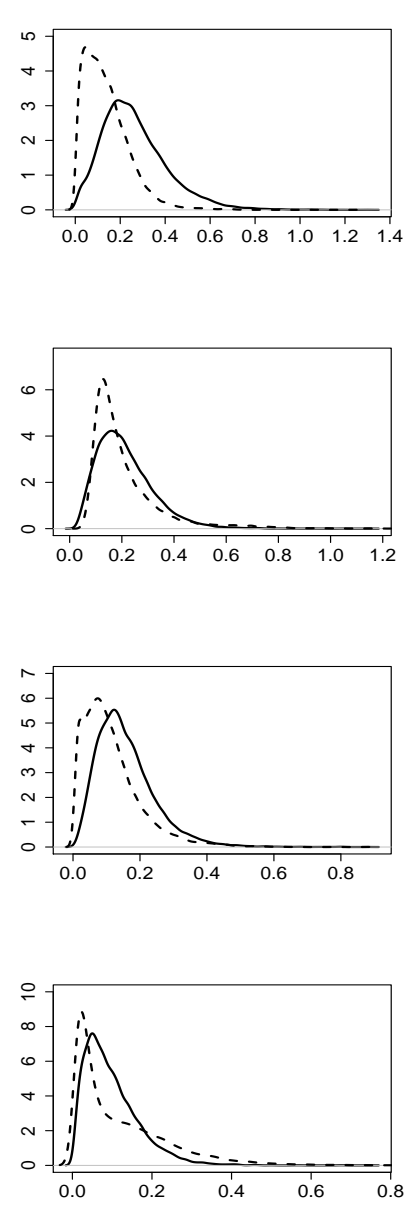

(c)
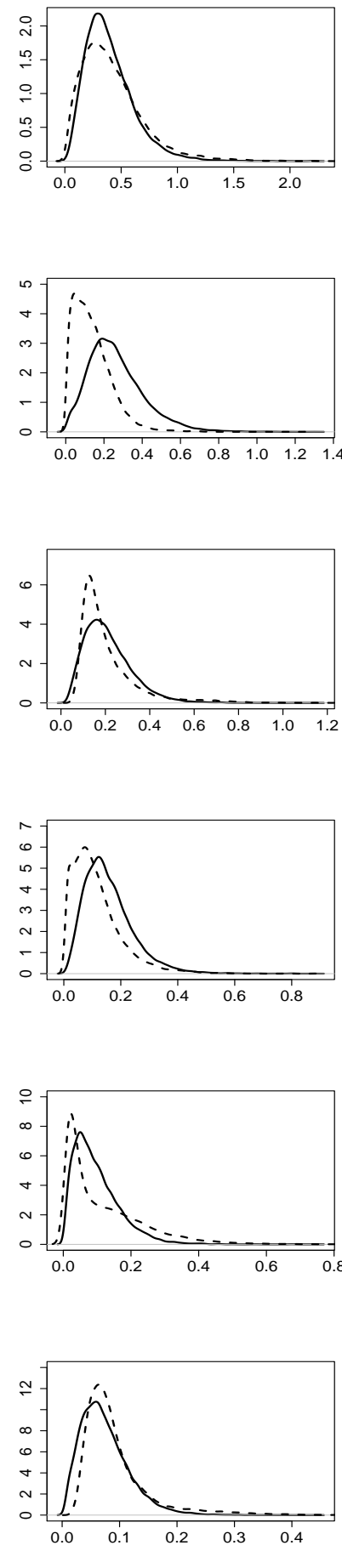

Figure A.1. Trace plots and posterior density plots obtained for $c_{5}, c_{6}, c_{8}, c_{9}, c_{10}$ and $c_{12}$ from the output of (a) the innovation scheme (solid line in (c)) and (b) the simulation filter (dashed line in (c)), using dataset $\mathcal{D}_{1}$ and $m=2$. 
(a)
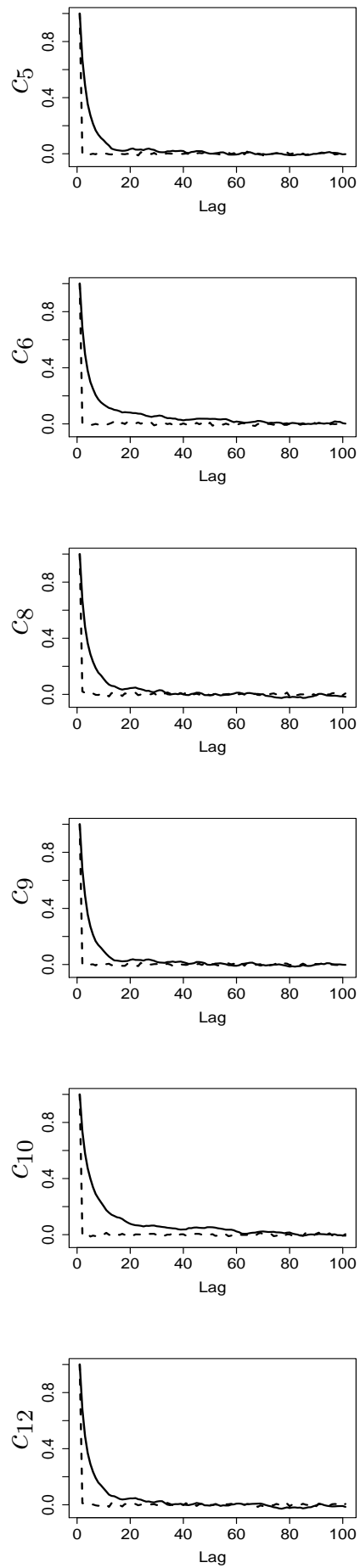

(b)
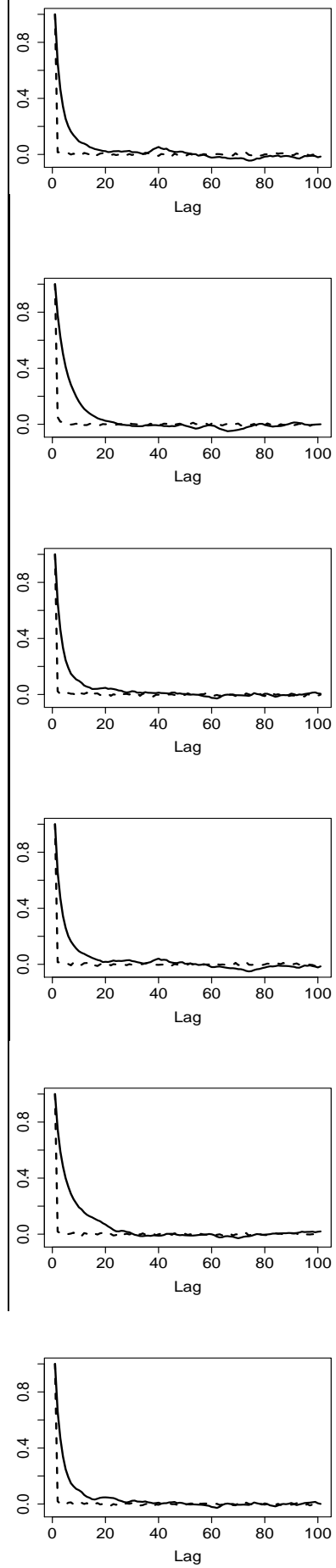

(c)
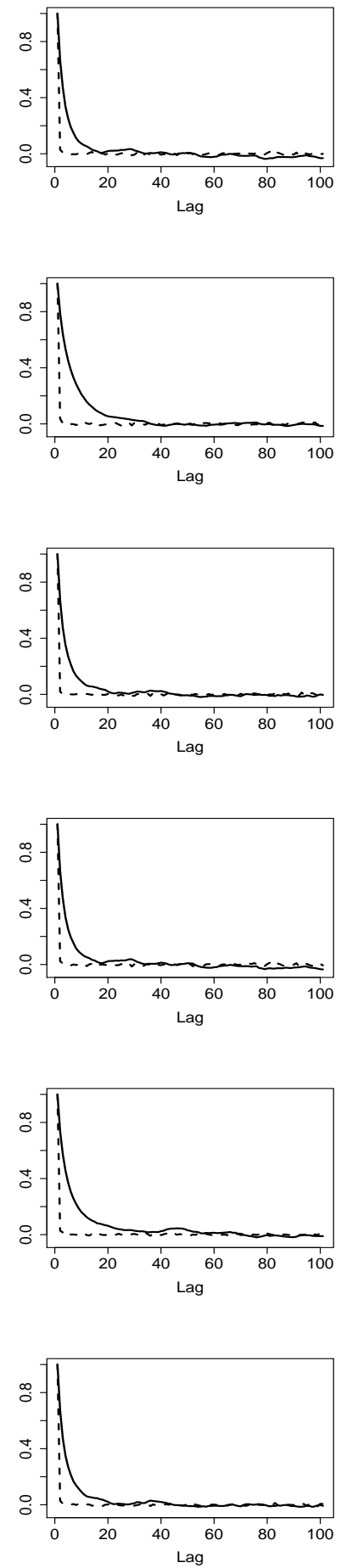

Figure A.2. Autocorrelation plots for $c_{5}, c_{6}, c_{8}, c_{9}, c_{10}$ and $c_{12}$ from the output of the innovation scheme (solid line) and the simulation filter (dashed line) using dataset $\mathcal{D}_{1}$ and (a) $m=2$, (b) $m=8$ and (c) $m=20$. 
(a)
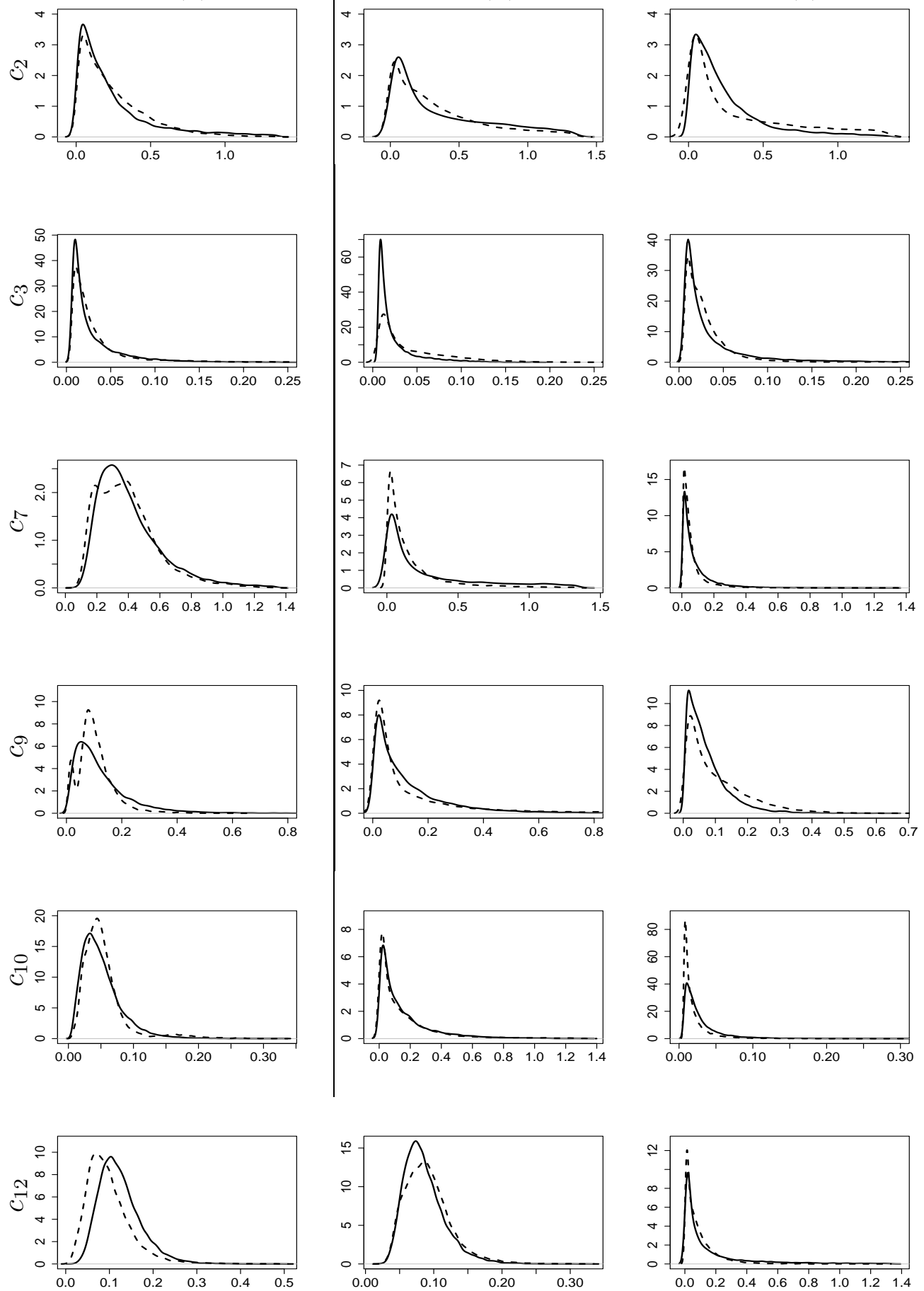

Figure A.3. Posterior density plots for $c_{2}, c_{3}, c_{7}, c_{9}, c_{10}$ and $c_{12}$ from the output of the innovation scheme (solid line) and the simulation filter (dashed line) using datasets (a) $\mathcal{D}_{2}$, (b) $\mathcal{D}_{3}$ and (c) $\mathcal{D}_{4}$. 
(a)
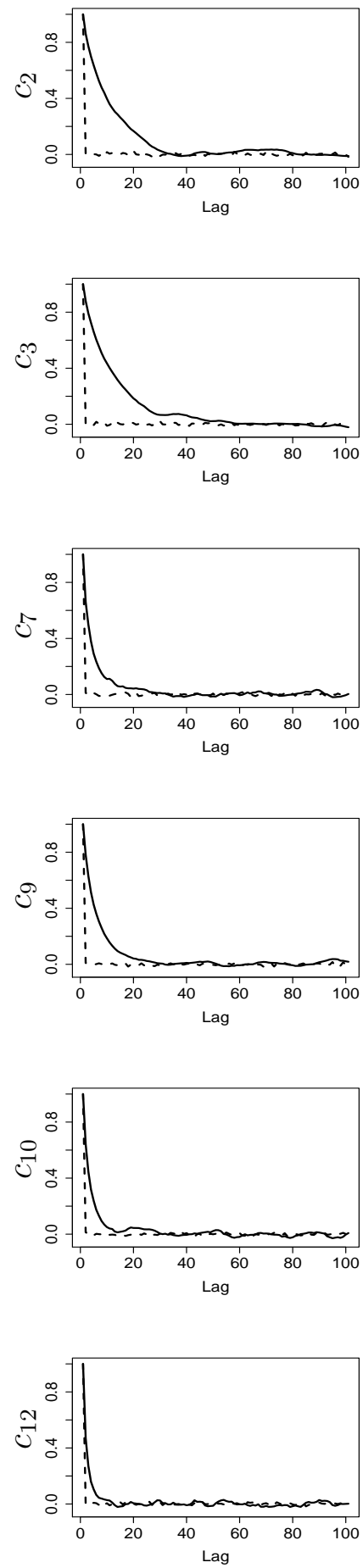

(b)
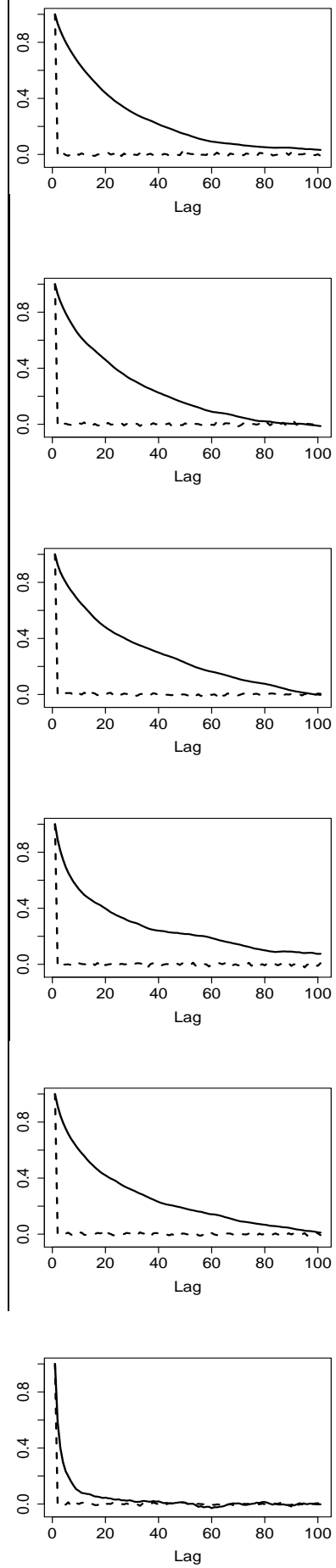

(c)
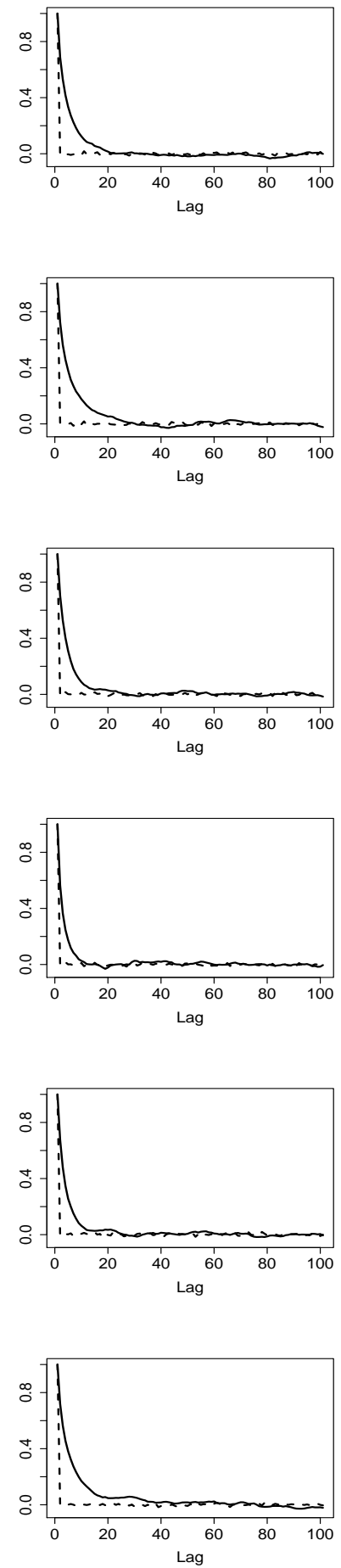

Figure A.4. Autocorrelation plots for $c_{2}, c_{3}, c_{7}, c_{9}, c_{10}$ and $c_{12}$ from the output of the innovation scheme (solid line) and the simulation filter (dashed line) using datasets (a) $\mathcal{D}_{2}$, (b) $\mathcal{D}_{3}$ and (c) $\mathcal{D}_{4}$. 


\section{List of Tables}

A.1 Posterior means and standard deviations for $\Theta$ estimated on 30 observations $\left(\mathcal{D}_{1}\right)$ from the fully observed model. Estimation results are based on a final sample of size 20,000, thinned from $4,000,000$ iterations of the innovation scheme.

A.2 Posterior means and standard deviations for $\Theta$ estimated on 30 observations $\left(\mathcal{D}_{1}\right)$ from the fully observed model. Estimation results are based on a final sample of size 30,000, thinned from $4,500,000$ iterations of the simulation filter.

A.3 Posterior means and standard deviations for parameters estimated using datasets $\mathcal{D}_{2}, \mathcal{D}_{3}$ and $\mathcal{D}_{4}$ from the partially observed model. Discretization is set at $m=20$ and the estimation results are based on a final sample of size 20,000, thinned from 4,000,000 iterations of the innovation scheme.

A.4 Posterior means and standard deviations for parameters estimated using datasets $\mathcal{D}_{2}, \mathcal{D}_{3}$ and $\mathcal{D}_{4}$ from the partially observed model. Discretization is set at $m=20$ and the estimation results are based on a final sample of size 20,000, thinned from 4,000,000 iterations of the simulation filter. 


\begin{tabular}{llccc}
\hline Parameter & True Value & \multicolumn{3}{c}{ Mean (Standard Deviation) } \\
\hline & & $m=2$ & $m=8$ & $m=20$ \\
$c_{1}$ & 0.08 & $0.018(0.011)$ & $0.030(0.030)$ & $0.036(0.039)$ \\
$c_{2}$ & 0.82 & $0.172(0.118)$ & $0.295(0.303)$ & $0.346(0.377)$ \\
$c_{1} / c_{2}$ & 0.096 & $0.118(0.057)$ & $0.122(0.076)$ & $0.119(0.068)$ \\
$c_{3}$ & 0.09 & $0.015(0.010)$ & $0.035(0.043)$ & $0.046(0.061)$ \\
$c_{4}$ & 0.9 & $0.118(0.091)$ & $0.283(0.371)$ & $0.379(0.507)$ \\
$c_{3} / c_{4}$ & 0.1 & $0.157(0.104)$ & $0.164(0.124)$ & $0.157(0.111)$ \\
$c_{5}$ & 0.25 & $0.398(0.219)$ & $0.447(0.256)$ & $0.476(0.270)$ \\
$c_{6}$ & 0.1 & $0.264(0.146)$ & $0.328(0.270)$ & $0.362(0.317)$ \\
$c_{7}$ & 0.35 & $0.361(0.218)$ & $0.353(0.294)$ & $0.377(0.297)$ \\
$c_{8}$ & 0.3 & $0.212(0.108)$ & $0.235(0.124)$ & $0.241(0.126)$ \\
$c_{9}$ & 0.1 & $0.158(0.087)$ & $0.176(0.101)$ & $0.187(0.106)$ \\
$c_{10}$ & 0.1 & $0.098(0.069)$ & $0.127(0.093)$ & $0.135(0.093)$ \\
$c_{11}$ & 0.12 & $0.411(0.238)$ & $0.436(0.536)$ & $0.419(0.591)$ \\
$c_{12}$ & 0.1 & $0.074(0.043)$ & $0.084(0.047)$ & $0.086(0.050)$ \\
$\sigma$ & 1.732 & $1.677(0.231)$ & $1.622(0.256)$ & $1.604(0.250)$ \\
\hline $\mathrm{A} 1$ & & & &
\end{tabular}

Table A.1

Posterior means and standard deviations for $\Theta$ estimated on 30 observations $\left(\mathcal{D}_{1}\right)$ from the fully observed model. Estimation results are based on a final sample of size 20,000, thinned from 4,000,000 iterations of the innovation scheme. 


\begin{tabular}{llccc}
\hline Parameter & True Value & \multicolumn{3}{c}{ Mean (Standard Deviation) } \\
\hline & & $m=2$ & $m=8$ & $m=20$ \\
$c_{1}$ & 0.08 & $0.021(0.017)$ & $0.025(0.016)$ & $0.042(0.038)$ \\
$c_{2}$ & 0.82 & $0.279(0.249)$ & $0.263(0.237)$ & $0.498(0.553)$ \\
$c_{1} / c_{2}$ & 0.096 & $0.097(0.058)$ & $0.111(0.078)$ & $0.102(0.075)$ \\
$c_{3}$ & 0.09 & $0.012(0.010)$ & $0.036(0.048)$ & $0.052(0.042)$ \\
$c_{4}$ & 0.9 & $0.100(0.113)$ & $0.310(0.547)$ & $0.509(0.521)$ \\
$c_{3} / c_{4}$ & 0.1 & $0.118(0.106)$ & $0.138(0.156)$ & $0.122(0.125)$ \\
$c_{5}$ & 0.25 & $0.418(0.276)$ & $0.407(0.479)$ & $0.435(0.321)$ \\
$c_{6}$ & 0.1 & $0.138(0.101)$ & $0.385(0.373)$ & $0.369(0.243)$ \\
$c_{7}$ & 0.35 & $0.359(0.101)$ & $0.392(0.447)$ & $0.301(0.201)$ \\
$c_{8}$ & 0.3 & $0.210(0.140)$ & $0.246(0.189)$ & $0.202(0.089)$ \\
$c_{9}$ & 0.1 & $0.114(0.086)$ & $0.108(0.148)$ & $0.127(0.105)$ \\
$c_{10}$ & 0.1 & $0.119(0.121)$ & $0.159(0.142)$ & $0.105(0.074)$ \\
$c_{11}$ & 0.12 & $0.275(0.144)$ & $0.372(0.503)$ & $0.389(0.299)$ \\
$c_{12}$ & 0.1 & $0.093(0.060)$ & $0.111(0.080)$ & $0.094(0.042)$ \\
$\sigma$ & 1.732 & $1.672(0.310)$ & $1.660(0.254)$ & $1.672(0.268)$ \\
\hline$A$ & & & &
\end{tabular}

Table A.2

Posterior means and standard deviations for $\Theta$ estimated on 30 observations $\left(\mathcal{D}_{1}\right)$ from the fully observed model. Estimation results are based on a final sample of size 30,000, thinned from 4,500,000 iterations of the simulation filter. 


\begin{tabular}{llccc}
\hline Parameter & True Value & \multicolumn{3}{c}{ Mean $($ Standard Deviation) } \\
\hline & & $\mathcal{D}_{2}$ & $\mathcal{D}_{3}$ & $\mathcal{D}_{4}$ \\
$c_{1}$ & 0.08 & $0.033(0.034)$ & $0.039(0.035)$ & $0.034(0.046)$ \\
$c_{2}$ & 0.82 & $0.243(0.254)$ & $0.374(0.362)$ & $0.247(0.250)$ \\
$c_{1} / c_{2}$ & 0.096 & $0.225(0.251)$ & $0.183(0.200)$ & $0.273(0.356)$ \\
$c_{3}$ & 0.09 & $0.031(0.033)$ & $0.025(0.024)$ & $0.038(0.048)$ \\
$c_{4}$ & 0.9 & $0.208(0.251)$ & $0.226(0.261)$ & $0.208(0.256)$ \\
$c_{3} / c_{4}$ & 0.1 & $0.341(0.367)$ & $0.214(0.211)$ & $0.450(0.670)$ \\
$c_{5}$ & 0.25 & $0.328(0.226)$ & $0.345(0.371)$ & $0.363(0.204)$ \\
$c_{6}$ & 0.1 & $0.225(0.173)$ & $0.137(0.212)$ & $0.076(0.142)$ \\
$c_{7}$ & 0.35 & $0.408(0.206)$ & $0.212(0.344)$ & $0.089(0.118)$ \\
$c_{8}$ & 0.3 & $0.345(0.101)$ & $0.629(0.299)$ & $0.293(0.338)$ \\
$c_{9}$ & 0.1 & $0.123(0.100)$ & $0.131(0.150)$ & $0.073(0.064)$ \\
$c_{10}$ & 0.1 & $0.050(0.030)$ & $0.157(0.182)$ & $0.029(0.029)$ \\
$c_{11}$ & 0.12 & $0.310(0.240)$ & $0.108(0.142)$ & $0.126(0.196)$ \\
$c_{12}$ & 0.1 & $0.124(0.048)$ & $0.086(0.030)$ & $0.157(0.233)$ \\
\hline $\mathrm{A} .3$ & & & &
\end{tabular}

Table A.3

Posterior means and standard deviations for parameters estimated using datasets $\mathcal{D}_{2}, \mathcal{D}_{3}$ and $\mathcal{D}_{4}$ from the partially observed model. Discretization is set at $m=20$ and the estimation results are based on a final sample of size 20,000, thinned from $4,000,000$ iterations of the innovation scheme. 


\begin{tabular}{llccc}
\hline Parameter & True Value & \multicolumn{3}{c}{ Mean $($ Standard Deviation) } \\
\hline & & $\mathcal{D}_{2}$ & $\mathcal{D}_{3}$ & $\mathcal{D}_{4}$ \\
$c_{1}$ & 0.08 & $0.020(0.014)$ & $0.037(0.031)$ & $0.036(0.058)$ \\
$c_{2}$ & 0.82 & $0.250(0.225)$ & $0.341(0.325)$ & $0.210(0.267)$ \\
$c_{1} / c_{2}$ & 0.096 & $0.204(0.354)$ & $0.248(0.341)$ & $0.326(0.432)$ \\
$c_{3}$ & 0.09 & $0.035(0.042)$ & $0.033(0.041)$ & $0.036(0.051)$ \\
$c_{4}$ & 0.9 & $0.228(0.323)$ & $0.321(0.342)$ & $0.184(0.237)$ \\
$c_{3} / c_{4}$ & 0.1 & $0.417(0.473)$ & $0.268(0.344)$ & $0.462(0.691)$ \\
$c_{5}$ & 0.25 & $0.348(0.272)$ & $0.326(0.355)$ & $0.396(0.263)$ \\
$c_{6}$ & 0.1 & $0.179(0.074)$ & $0.061(0.107)$ & $0.071(0.112)$ \\
$c_{7}$ & 0.35 & $0.393(0.194)$ & $0.174(0.225)$ & $0.120(0.183)$ \\
$c_{8}$ & 0.3 & $0.241(0.072)$ & $0.556(0.270)$ & $0.373(0.389)$ \\
$c_{9}$ & 0.1 & $0.103(0.064)$ & $0.162(0.241)$ & $0.076(0.066)$ \\
$c_{10}$ & 0.1 & $0.054(0.037)$ & $0.156(0.207)$ & $0.031(0.032)$ \\
$c_{11}$ & 0.12 & $0.198(0.189)$ & $0.077(0.062)$ & $0.151(0.176)$ \\
$c_{12}$ & 0.1 & $0.098(0.052)$ & $0.092(0.034)$ & $0.159(0.258)$ \\
\hline $\mathrm{A} .4$ & & & &
\end{tabular}

Table A.4

Posterior means and standard deviations for parameters estimated using datasets $\mathcal{D}_{2}, \mathcal{D}_{3}$ and $\mathcal{D}_{4}$ from the partially observed model. Discretization is set at $m=20$ and the estimation results are based on a final sample of size 20,000, thinned from $4,000,000$ iterations of the simulation filter. 